\title{
Stability and Synchronization of Discrete-Time Markovian Jumping Neural Networks With Mixed Mode-Dependent Time Delays
}

\author{
Yurong Liu, Zidong Wang, Senior Member, IEEE, Jinling Liang, and Xiaohui Liu
}

\begin{abstract}
In this paper, we introduce a new class of discretetime neural networks (DNNs) with Markovian jumping parameters as well as mode-dependent mixed time delays (both discrete and distributed time delays). Specifically, the parameters of the DNNs are subject to the switching from one to another at different times according to a Markov chain, and the mixed time delays consist of both discrete and distributed delays that are dependent on the Markovian jumping mode. We first deal with the stability analysis problem of the addressed neural networks. A special inequality is developed to account for the mixed time delays in the discrete-time setting, and a novel Lyapunov-Krasovskii functional is put forward to reflect the mode-dependent time delays. Sufficient conditions are established in terms of linear matrix inequalities (LMIs) that guarantee the stochastic stability. We then turn to the synchronization problem among an array of identical coupled Markovian jumping neural networks with mixed mode-dependent time delays. By utilizing the Lyapunov stability theory and the Kronecker product, it is shown that the addressed synchronization problem is solvable if several LMIs are feasible. Hence, different from the commonly used matrix norm theories (such as the $M$-matrix method), a unified LMI approach is developed to solve the stability analysis and synchronization problems of the class of neural networks under investigation, where the LMIs can be easily solved by using the available Matlab LMI toolbox. Two numerical examples are presented to illustrate the usefulness and effectiveness of the main results obtained.
\end{abstract}

Index Terms-Discrete-time neural networks (DNNs), linear matrix inequality, Markovian jumping parameters, mixed time delays, stochastic stability, synchronization.

Manuscript received March 17, 2008; revised January 21, 2009; accepted February 16, 2009. First published May 26, 2009; current version published July 09, 2009. This work was supported in part by the Biotechnology and Biological Sciences Research Council (BBSRC) of the U.K. under Grants BB/C506264/1 and 100/EGM17735, by the Engineering and Physical Sciences Research Council (EPSRC) of the U.K. under Grants GR/S27658/01 and EP/C524586/1, by an International Joint Project sponsored by the Royal Society of the U.K., by the National Natural Science Foundation of China under Grants 60774073 and 60804028, by the Natural Science Foundation of Jiangsu Province of China under Grant BK2007075, by the Specialized Research Fund for the Doctoral Program of Higher Education for New Teachers under Grant 200802861044, by the Teaching and Research Fund for Excellent Young Teachers at Southeast University of China, and by the Alexander von Humboldt Foundation of Germany.

Y. Liu is with the Department of Mathematics, Yangzhou University, Yangzhou 225002, China (e-mail: liuyurong@gmail.com).

Z. Wang and X. Liu are with the Department of Information Systems and Computing, Brunel University, Uxbridge, Middlesex UB8 3PH, U.K. (e-mail: Zidong.Wang@brunel.ac.uk).

J. Liang is with the Department of Mathematics, Southeast University, Nanjing 210096, China (e-mail: jinlliang@gmail.com).

Color versions of one or more of the figures in this paper are available online at http://ieeexplore.ieee.org.

Digital Object Identifier 10.1109/TNN.2009.2016210

\section{INTRODUCTION}

$\mathbf{T}$ HE last few decades have seen successful applications of recurrent neural networks (RNNs) to a variety of areas including pattern recognition, associative memory, and combinational optimization [1], [31], [32]. Among many dynamical behaviors of the neural networks, stability and synchronization are two of the most important ones that have received considerable research attention. For instance, if a neural network is employed to solve some optimization problems, it is highly desirable for the neural network to have a unique globally stable equilibrium, and it is not surprising that the stability analysis of neural networks has been an ever hot research topic resulting in enormous stability conditions reported in the literature. On the other hand, the synchronization in coupled identical delayed neural networks has been shown to have an important impact on the fundamental science (e.g., the self-organization behavior in the brain). The synchronization hypothesis for brain activities has been confirmed by modern neurophysiological experiments (such as olfactory bulb and cortex, visual cortex, hippocampus, neocortex, and thalamo-cortical systems) [12], [17]. In [9], the Lyapunov functional method and the Hermitian matrix theory have been employed to investigate the global synchronization of the coupled neural networks by defining a distance between any point and the synchronization manifold. For more details concerning synchronization of neural networks, see [5], [24]-[27], [42], and the references cited therein.

Time delay exhibits as a typical characteristic of signal transmission between neurons, and therefore, becomes one of the main sources for causing instability and poor performances of neural networks (see, e.g., [1] and [13]-[15]). Time delays can be generally categorized as two types: discrete and distributed. Discrete time delay seems easy to be identified in practice and, therefore, stability analysis for RNNs with discrete delays has been an attractive subject of research in the past few years. Various sufficient conditions, either delay-dependent or delay-independent, have been proposed to guarantee the global asymptotic or exponential stability for the RNNs; see, e.g., [6], [34], [38], [39], and [41] for some recent publications. Distributed delays are due primarily to the presence of an amount of parallel pathways of a variety of axon sizes and lengths, which provides a neural network with a spatial nature. Distributed delays over a certain duration of time have been introduced in [30] and [36] such that the distant past has less influence compared to the recent behavior of the state. Recently, the global stability analysis problem for general RNNs with mixed time delays (also called 
discrete and distributed delays) has received an increasing research attention and many relevant results have been reported in the literature; see, e.g., [21], [38], [39], [41], and the references therein.

It is quite common for RNNs to have problems in catching long-term dependencies in the input stream. This is referred to as the information latching problem [2] that can be handled by extracting finite-state representations (also called clusters, patterns, or modes) from trained networks [3], [7], [8], [11]. Recently, it has been shown in [37] that the switching (or jumping) between different RNN modes can be governed by a Markovian chain. Hence, an RNN with such a "jumping" character may switch from one to another according to Markovian chain, and therefore, RNNs with Markovian jumping parameters are of great significance in modeling a class of neural networks with finite network modes. Dynamics analysis problem of Markovian jumping RNNs has recently stirred initial research interests. For example, in [40], the exponential stability problem has been first addressed for a class of delayed recurrent neural networks with Markovian jumping parameters. In [44], a delay-dependent approach has been developed to deal with the stability analysis problem for Markovian jumping stochastic Cohen-Grossberg neural networks with mixed time delays. In [33], a noise-induced stabilization method has been proposed for RNNs with mixed time-varying delays and Markovian switching parameters.

It is worth pointing out that, up to now, all the aforementioned results concerning dynamics analysis problems for delayed RNNs with or without Markovian jumping parameters have been applied to continuous-time models. In implementing and applications of neural networks, however, discrete-time neural networks play a more crucial role than their continuous-time counterparts in today's digital world. In order to investigate the dynamical characteristics with respect to digital signal transmission, it is usually essential to formulate the discrete-time analog [28], [35]. In the past few years, various stability criteria have been proposed for discrete-time neural networks (DNNs) in the literature; see, e.g., [18], [43], and [45] for DNNs without time delays and [10], [19], and [20] for DNNs with discrete time delays. Note that pioneering work has been carried out in [29] for preserving exponential stability in discrete-time analogs of artificial neural networks with distributed delays. In [25]-[27], Lu and Chen investigated the synchronization problem for an array of discrete-time coupled complex networks in a systematic way and obtained a series of elegant results by using innovative manifold/graph approaches. Unfortunately, so far, the stability analysis and synchronization problems for discrete-time Markovian jumping neural networks with or without mixed mode-dependent time delays have not been fully investigated yet and remains challenging. The major challenges are as follows: 1) how to represent the finitely distributed time delays in the discrete-time domain; 2) how to deal with mode-dependent distributed time delays; and 3) how to establish a unified framework to handle both the Markovian jumping parameters and mixed time delays. It is, therefore, the main purpose of this paper to make the first attempt to handle the listed challenges.

In this paper, we consider the stability analysis and synchronization problems for a new class of DNNs with Markovian jumping parameters as well as mode-dependent mixed time delays. Note that the mixed time delays comprise both the discrete and distributed delays that are dependent on the Markovian jumping mode. We first develop a special matrix inequality to account for the mixed time delays in the discrete-time setting, and then a novel Lyapunov-Krasovskii functional is proposed to reflect the mode-dependent nature of the time delays. A linear matrix inequality (LMI) approach is utilized to derive sufficient conditions guaranteeing the stochastic stability of the considered neural networks. The synchronization problem is then studied among an array of identical coupled Markovian jumping neural networks with mixed mode-dependent time delays, and it is shown that the addressed synchronization problem is solvable if several LMIs are feasible. Different from the commonly used matrix norm theories (such as the $M$-matrix method), a unified LMI approach is developed to solve both the stability analysis and synchronization problems of the class of neural networks under investigation, where the LMIs can be easily solved by using the available Matlab LMI toolbox [4]. Two numerical examples are presented to illustrate the usefulness and effectiveness of the main results obtained.

Notations: Throughout this paper, $\mathbb{R}^{n}$ and $\mathbb{R}^{n \times m}$ denote, respectively, the $n$-dimensional Euclidean space and the set of all $n \times m$ real matrices. The superscript " $T$ " denotes the transpose and the notation $X \geq Y$ (respectively, $X>Y$ ) where $X$ and $Y$ are symmetric matrices, means that $X-Y$ is positive semidefinite (respectively, positive definite); $I$ is the identity matrix with compatible dimension. $|\cdot|$ refers to the Euclidean vector norm; the notation $A \otimes B$ stands for the Kronecker product of matrices $A$ and $B$. If $A$ is a matrix, $\lambda_{\min }(\cdot)$ and $\lambda_{\max }(\cdot)$ denote the minimum and the maximum eigenvalue, respectively. In symmetric block matrices, we use an asterisk " $*$ " to represent a term that is induced by symmetry and $\operatorname{diag}\{\ldots\}$ stands for a block-diagonal matrix. $\mathbb{E}[x]$ and $\mathbb{E}[x \mid y]$ will, respectively, mean the expectation of $x$ and the expectation of $x$ conditional on $y$. Matrices, if their dimensions are not explicitly stated, are assumed to be compatible for algebraic operations.

\section{PROBLEM FORMULATION}

In this section, we will introduce the model of neural networks, give the definition of the stability related, and put forward the problem to be dealt with in this paper.

Let $r(k)(k \geq 0)$ be a Markov chain taking values in a finite state space $\mathcal{S}=\{1,2, \ldots, N\}$ with probability transition matrix $\boldsymbol{\Pi}=\left(\pi_{i j}\right)_{N \times N}$ given by

$$
\mathcal{P}\{r(k+1)=j \mid r(k)=i\}=\pi_{i j} \quad \forall i, j \in \mathcal{S}
$$

where $\pi_{i j} \geq 0(i, j \in \mathcal{S})$ is the transition probability from $i$ to $j$ and $\sum_{j=1}^{N} \pi_{i j}=1, \forall i \in \mathcal{S}$.

Consider a discrete-time $n$-neuron neural network with $N$ modes described by the following dynamical equation:

$$
\begin{aligned}
x(k+1)= & D(r(k)) x(k)+A(r(k)) F(x(k))+B(r(k)) \\
& \times G\left(x\left(k-\tau_{1, r(k)}\right)\right)+C(r(k)) \sum_{v=1}^{\tau_{2, r(k)}} H(x(k-v))
\end{aligned}
$$

$$
x(s)=\phi(s), \quad s=-\tau,-\tau+1, \ldots,-1,0
$$


where $x(k)=\left(x_{1}(k), x_{2}(k), \ldots, x_{n}(k)\right)^{T}$ is the neural state vector; the constant matrices $D(r(k))=\operatorname{diag}\left\{d_{1}(r(k))\right.$, $\left.d_{2}(r(k)), \ldots, d_{n}(r(k))\right\}$ describe the rate with which the each neuron will reset its potential to the resting state in isolation when disconnected from the networks and external inputs; $A(r(k))=\left[a_{i j}(r(k))\right]_{n \times n}, B(r(k))=\left[b_{i j}(r(k))\right]_{n \times n}$ and $C(r(k))=\left[c_{i j}(r(k))\right]_{n \times n}$ are, respectively, the connection weight matrix, the delayed connection weight matrix, and the distributively delayed connection weight matrix; $\tau_{1, r(k)}$ denotes the discrete time delay in mode $r(k)$ while $\tau_{2, r(k)}$ describes the distributed time delay, and both of them are mode dependent. In (1a), $F(x(k))=\left(f_{1}\left(x_{1}(k)\right), f_{2}\left(x_{2}(k)\right), \ldots, f_{n}\left(x_{n}(k)\right)\right)^{T}$, $G\left(x\left(k-\tau_{1, r(k)}\right)\right)=\left(g_{1}\left(x_{1}\left(k-\tau_{1, r(k)}\right)\right), g_{2}\left(x_{2}\left(k-\tau_{1, r(k)}\right)\right)\right.$, $\left.\ldots, g_{n}\left(x_{n}\left(k-\tau_{1, r(k)}\right)\right)\right)^{T}$, and $H(x(k))=\left(h_{1}\left(x_{1}(k)\right)\right.$, $\left.h_{2}\left(x_{2}(k)\right), \ldots, h_{n}\left(x_{n}(k)\right)\right)^{T}$ are the nonlinear activation functions; and $\phi(s)$ describes the initial condition.

In the sequel, let $\tau=\max \left\{\bar{\tau}_{1}, \bar{\tau}_{2}\right\}$ with $\bar{\tau}_{1}=\max \left\{\tau_{1, i} \mid i \in \mathcal{S}\right\}$ and $\bar{\tau}_{2}=\max \left\{\tau_{2, i} \mid i \in \mathcal{S}\right\}$. Furthermore, we denote $\underline{\tau}_{1}=$ $\min \left\{\tau_{1, i} \mid i \in \mathcal{S}\right\}, \underline{\tau}_{2}=\min \left\{\tau_{2, i} \mid i \in \mathcal{S}\right\}$, and $\underline{\pi}=\min \left\{\pi_{i i} \mid i \in \mathcal{S}\right\}$.

Remark 1: The discrete-time recurrent neural network (1a) can be considered as a discrete analog of the continuous-time recurrent neural network of the form

$$
\begin{aligned}
\frac{d x}{d t}= & D(r(t)) x(t)+A(r(t)) F(x(t))+B(r(t)) \\
& \times G\left(x\left(t-\tau_{1, r(t)}\right)\right)+C(r(t)) \int_{t-\tau_{2, r(t)}}^{t} H(x(s)) d s
\end{aligned}
$$

where $r(t)$ denotes a continuous-time Markov process, and both $\tau_{1, r(t)}$ and $\tau_{2, r(t)}$, describing discrete and distributed time delays, respectively, are mode dependent as well. For the continuous-time case, the neural networks with distributed time delay first appeared in [16], and has now been studied extensively. On the other hand, the neural networks with both discrete and distributed delays are receiving considerable research attention [21].

Usually, a neural network model contains a constant vector representing the external bias on the neurons [21], [22]. However, there is a standard way to shift the system equilibrium point to the origin, and the constant vector will disappear accordingly. Therefore, for the sake of simplicity, such an external bias is dropped in (1a) without loss of generality.

Throughout this paper, we make the following assumptions.

Assumption 1: $F(\cdot), G(\cdot)$, and $H(\cdot)$ are bounded functions and satisfy $F(0)=G(0)=H(0)=0$.

Assumption 2 [21], [22]: For the activation functions $F(\cdot)$, $G(\cdot)$, and $H(\cdot)$, there exist constants $\lambda_{i}^{-}, \lambda_{i}^{+}, \sigma_{i}^{-}, \sigma_{i}^{+}, v_{i}^{-}$, and $v_{i}^{+}$such that

$$
\begin{gathered}
\lambda_{i}^{-} \leq \frac{f_{i}\left(s_{1}\right)-f_{i}\left(s_{2}\right)}{s_{1}-s_{2}} \leq \lambda_{i}^{+} \\
\sigma_{i}^{-} \leq \frac{g_{i}\left(s_{1}\right)-g_{i}\left(s_{2}\right)}{s_{1}-s_{2}} \leq \sigma_{i}^{+} \\
v_{i}^{-} \leq \frac{h_{i}\left(s_{1}\right)-h_{i}\left(s_{2}\right)}{s_{1}-s_{2}} \leq v_{i}^{+} .
\end{gathered}
$$

Remark 2: As pointed out in [21] and [22], the constants $\lambda_{i}^{-}$, $\lambda_{i}^{+}, \sigma_{i}^{-}, \sigma_{i}^{+}, v_{i}^{-}$, and $v_{i}^{+}$in Assumption 2 are allowed to be positive, negative, or zero. Hence, the resulting activation functions could be nonmonotonic, and are more general than the usual sigmoid functions and the recently commonly used Lipschitz conditions. Such a description is very precise/tight in quantifying the lower and upper bounds of the activation functions, hence very helpful for using LMI-based approach to reduce the possible conservatism.

Definition 1: Neural network (1) is said to be asymptotically stable in the mean square if, for any solution $x(k)$ of (1), the following holds:

$$
\lim _{k \rightarrow \infty} \mathbb{E}\left[|x(k)|^{2}\right]=0 .
$$

Furthermore, neural network (1) is said to be exponentially stable in the mean square if, for any solution $x(k)$ of (1), there exist constants $0<\beta<1$ and $\mu>0$ such that

$$
\mathbb{E}\left[|x(k)|^{2}\right] \leq \mu \beta^{k} \quad \forall k>0 .
$$

In this paper, we will first deal with the stochastic stability problem for the system (1), and then turn to the synchronization problem between the array of identical neural networks. By constructing new Lyapunov-Krasovskii functionals, sufficient conditions for the stability and synchronization problems are derived. These criteria are expressed in the form of LMIs, which are easily checked by using the Matlab LMI toolbox.

\section{StOchastic STABILITY}

This section is devoted to the stability analysis for system (1). To start with, we introduce some lemmas to be used in deriving our results.

Lemma 1 [21]: Let $X$ and $Y$ be any $n$-dimensional real vectors and $P$ be an $n \times n$ symmetric positive-semidefinite matrix. Then, the following matrix inequality holds:

$$
2 X^{T} P Y \leq X^{T} P X+Y^{T} P Y .
$$

Lemma 2 [23]: Let $M \in \mathbb{R}^{n \times n}$ be a symmetric positivesemidefinite matrix, $\mathbf{x}_{i} \in \mathbb{R}^{n}$ be a vector, and $a_{i} \geq 0(i=$ $1,2, \ldots, m)$ be scalars, where $m$ is a positive integer. Then, the following inequality holds:

$$
\left(\sum_{i=1}^{m} a_{i} \mathbf{x}_{i}\right)^{T} M\left(\sum_{i=1}^{m} a_{i} \mathbf{x}_{i}\right) \leq\left(\sum_{i=1}^{m} a_{i}\right) \sum_{i=1}^{m} a_{i} \mathbf{x}_{i}^{T} M \mathbf{x}_{i} .
$$

Lemma 3: Suppose that $\mathcal{B}=\operatorname{diag}\left\{\beta_{1}, \beta_{2}, \ldots, \beta_{n}\right\}$ is a positive-semidefinite diagonal matrix. Let $x=\left(x_{1}, x_{2}, \ldots, x_{n}\right)^{T} \in$ $\mathbb{R}^{n}$, and $\mathcal{H}(x)=\left(\hbar_{1}\left(x_{1}\right), \hbar_{2}\left(x_{2}\right), \ldots, \hbar_{n}\left(x_{n}\right)\right)^{T}$ be a continuous nonlinear function satisfying

$l_{i}^{-} \leq \frac{\hbar_{i}(s)}{s} \leq l_{i}^{+}, \quad s \neq 0, \quad s \in \mathbb{R}, \quad i=1,2, \ldots, n$

with $l_{i}^{-}$and $l_{i}^{+}$being constant scalars. Then

$$
\left[\begin{array}{c}
x \\
\mathcal{H}(x)
\end{array}\right]^{T}\left[\begin{array}{cc}
\mathcal{B} L_{1} & -\mathcal{B} L_{2} \\
-\mathcal{B} L_{2} & \mathcal{B}
\end{array}\right]\left[\begin{array}{c}
x \\
\mathcal{H}(x)
\end{array}\right] \leq 0
$$

or

$$
x^{T} \mathcal{B} L_{1} x-2 x^{T} \mathcal{B} L_{2} \mathcal{H}(x)+\mathcal{H}^{T}(x) \mathcal{B H}(x) \leq 0
$$


where $L_{1}=\operatorname{diag}\left(l_{1}^{+} l_{1}^{-}, l_{2}^{+} l_{2}^{-}, \ldots, l_{n}^{+} l_{n}^{-}\right)$and $L_{2}=$ $\operatorname{diag}\left(\left(l_{1}^{+}+l_{1}^{-}\right) / 2,\left(l_{2}^{+}+l_{2}^{-}\right) / 2, \ldots,\left(l_{n}^{+}+l_{n}^{-}\right) / 2\right)$.

Proof: Notice that (7) is equivalent to

$$
\left(\hbar_{i}\left(x_{i}\right)-l_{i}^{+} x_{i}\right)\left(\hbar_{i}\left(x_{i}\right)-l_{i}^{-} x_{i}\right) \leq 0
$$

or

$$
\left[\begin{array}{c}
x \\
\mathcal{H}(x)
\end{array}\right]^{T}\left[\begin{array}{cc}
l_{i}^{+} l_{i}^{-} e_{i} e_{i}^{T} & -\frac{l_{i}^{+}+l_{i}^{-}}{2} e_{i} e_{i}^{T} \\
-\frac{l_{i}^{+}+l_{i}^{-}}{2} e_{i} e_{i}^{T} & e_{i} e_{i}^{T}
\end{array}\right]\left[\begin{array}{c}
x \\
\mathcal{H}(x)
\end{array}\right] \leq 0
$$

where $e_{k}$ denotes the unit column vector having " 1 " element on its $k$ th row and zeros elsewhere.

Since $\beta_{i} \geq 0$, it follows readily that

$\sum_{i=1}^{n} \beta_{i}\left[\begin{array}{c}x \\ \mathcal{H}(x)\end{array}\right]^{T}\left[\begin{array}{cc}l_{i}^{+} l_{i}^{-} e_{i} e_{i}^{T} & -\frac{l_{i}^{+}+l_{i}^{-}}{2} e_{i} e_{i}^{T} \\ -\frac{l_{i}^{+}+l_{i}^{-}}{2} e_{i} e_{i}^{T} & e_{i} e_{i}^{T}\end{array}\right]\left[\begin{array}{c}x \\ \mathcal{H}(x)\end{array}\right] \leq 0$

namely

$$
\left[\begin{array}{c}
x \\
\mathcal{H}(x)
\end{array}\right]^{T}\left[\begin{array}{cc}
\mathcal{B} L_{1} & -\mathcal{B} L_{2} \\
-\mathcal{B} L_{2} & \mathcal{B}
\end{array}\right]\left[\begin{array}{c}
x \\
\mathcal{H}(x)
\end{array}\right] \leq 0
$$

This completes the proof of this lemma.

Lemma 4 (Schur Complement [4]): Given constant matrices $\Omega_{1}, \Omega_{2}$, and $\Omega_{3}$ where $\Omega_{1}=\Omega_{1}^{T}$ and $\Omega_{2}>0$, then

if and only if

$$
\Omega_{1}+\Omega_{3}^{T} \Omega_{2}^{-1} \Omega_{3}<0
$$

$$
\left[\begin{array}{cc}
\Omega_{1} & \Omega_{3}^{T} \\
\Omega_{3} & -\Omega_{2}
\end{array}\right]<0 .
$$

Lemma 5: Let $\mathcal{U}=\left(\alpha_{i j}\right)_{N \times N}, P \in \mathbb{R}^{n \times n}, x=$ $\left(x_{1}^{T}, x_{2}^{T}, \ldots, x_{N}^{T}\right)^{T}$, and $y=\left(y_{1}^{T}, y_{2}^{T}, \ldots, y_{N}^{T}\right)^{T}$ with $x_{k}, y_{k} \in \mathbb{R}^{n}(k=1,2, \ldots, N)$. If $\mathcal{U}=\mathcal{U}^{T}$ and the sum of entries in each row of $\mathcal{U}$ is zero, then

$$
x^{T}(\mathcal{U} \otimes P) y=-\sum_{1 \leq i<j \leq N} \alpha_{i j}\left(x_{i}-x_{j}\right)^{T} P\left(y_{i}-y_{j}\right) .
$$

Proof: According to the definition of Kronecker product of matrices, we have

$$
\begin{aligned}
x^{T}(\mathcal{U} \otimes P) y= & \sum_{i=1}^{N} \sum_{j=1}^{N} \alpha_{i j} x_{i}^{T} P y_{j} \\
= & \sum_{1 \leq i<j \leq N}^{N} \alpha_{i j} x_{i}^{T} P y_{j}+\sum_{1 \leq j<i \leq N} \alpha_{i j} x_{i}^{T} P y_{j} \\
& +\sum_{i=1}^{N} \alpha_{i i} x_{i}^{T} P y_{i} .
\end{aligned}
$$

Therefore

Since the sum of entries in each row of $\mathcal{U}$ is zero, then

$$
\begin{aligned}
\sum_{i=1}^{N} \alpha_{i i} x_{i}^{T} P y_{i} & =-\sum_{i=1}^{N} \sum_{j \neq i} \alpha_{i j} x_{i}^{T} P y_{i} \\
& =-\sum_{1 \leq i<j \leq N} \alpha_{i j} x_{i}^{T} P y_{i}-\sum_{1 \leq j<i \leq N} \alpha_{i j} x_{i}^{T} P y_{i} .
\end{aligned}
$$

$$
\begin{aligned}
& x^{T}(\mathcal{U} \otimes P) y \\
& =-\sum_{1 \leq i<j \leq N} \alpha_{i j} x_{i}^{T} P\left(y_{i}-y_{j}\right)+\sum_{1 \leq j<i \leq N} \alpha_{i j} x_{i}^{T} P\left(y_{j}-y_{i}\right) \\
& =-\sum_{1 \leq i<j \leq N} \alpha_{i j} x_{i}^{T} P\left(y_{i}-y_{j}\right)+\sum_{1 \leq i<j \leq N} \alpha_{j i} x_{j}^{T} P\left(y_{i}-y_{j}\right) \\
& =-\sum_{1 \leq i<j \leq N} \alpha_{i j} x_{i}^{T} P\left(y_{i}-y_{j}\right)+\sum_{1 \leq i<j \leq N} \alpha_{i j} x_{j}^{T} P\left(y_{i}-y_{j}\right) \\
& =-\sum_{1 \leq i<j \leq N} \alpha_{i j}\left(x_{i}-x_{j}\right)^{T} P\left(y_{i}-y_{j}\right) .
\end{aligned}
$$

Notice that here we have used the condition $\mathcal{U}=\mathcal{U}^{T}$.

Lemma 6: Let $A$ be a symmetric matrix and suppose that the sum of all elements in each row of $A$ is zero, then for any positive integer $n$, the sum of all elements in each row of $A^{n}$ is zero.

Proof: It can be verified easily by simple matrix operations.

Hereafter, we denote

$$
\begin{aligned}
& \Lambda_{1}=\operatorname{diag}\left\{\lambda_{1}^{+} \lambda_{1}^{-}, \lambda_{2}^{+} \lambda_{2}^{-}, \ldots, \lambda_{n}^{+} \lambda_{n}^{-}\right\} \\
& \Lambda_{2}=\operatorname{diag}\left\{\frac{\lambda_{1}^{+}+\lambda_{1}^{-}}{2}, \frac{\lambda_{2}^{+}+\lambda_{2}^{-}}{2}, \ldots, \frac{\lambda_{n}^{+}+\lambda_{n}^{-}}{2}\right\} \\
& \Sigma_{1}=\operatorname{diag}\left\{\sigma_{1}^{+} \sigma_{1}^{-}, \sigma_{2}^{+} \sigma_{2}^{-}, \ldots, \sigma_{n}^{+} \sigma_{n}^{-}\right\} \\
& \Sigma_{2}=\operatorname{diag}\left\{\frac{\sigma_{1}^{+}+\sigma_{1}^{-}}{2}, \frac{\sigma_{2}^{+}+\sigma_{2}^{-}}{2}, \ldots, \frac{\sigma_{n}^{+}+\sigma_{n}^{-}}{2}\right\} \\
& \Upsilon_{1}=\operatorname{diag}\left\{v_{1}^{+} v_{1}^{-}, v_{2}^{+} v_{2}^{-}, \ldots, v_{n}^{+} v_{n}^{-}\right\} \\
& \Upsilon_{2}=\operatorname{diag}\left\{\frac{v_{1}^{+}+v_{1}^{-}}{2}, \frac{v_{2}^{+}+v_{2}^{-}}{2}, \ldots, \frac{v_{n}^{+}+v_{n}^{-}}{2}\right\} .
\end{aligned}
$$

Now we state the main result in this section.

Theorem 1: Under Assumptions 1 and 2, the delayed neural network (1) is asymptotically stable in the mean square if there exist a set of matrices $P_{i}>0$, two matrices $Q>0$ and $R>0$, and three sets of diagonal matrices $\Omega_{i}>0, \Theta_{i}>0$, and $\Delta_{i}>0$ such that LMI (9) shown at the bottom of the page holds, where

$$
\begin{aligned}
& \bar{P}_{i}=\sum_{j=1}^{N} \pi_{i j} P_{j} \\
& \Pi_{i}=-P_{i}-\Omega_{i} \Lambda_{1}-\Theta_{i} \Sigma_{1}-\Delta_{i} \Upsilon_{1}
\end{aligned}
$$

$$
\Phi_{i}:=\left(\begin{array}{ccccccc}
\Pi_{i} & \Omega_{i} \Lambda_{2} & \Theta_{i} \Sigma_{2} & 0 & \Delta_{i} \Upsilon_{2} & 0 & D(i) \bar{P}_{i} \\
\Omega_{i} \Lambda_{2} & -\Omega_{i} & 0 & 0 & 0 & 0 & A^{T}(i) \bar{P}_{i} \\
\Theta_{i} \Sigma_{2} & 0 & \Xi_{i} & 0 & 0 & 0 & 0 \\
0 & 0 & 0 & -Q & 0 & 0 & B^{T}(i) \bar{P}_{i} \\
\Delta_{i} \Upsilon_{2} & 0 & 0 & 0 & \hat{\alpha}_{i} R-\Delta_{i} & 0 & 0 \\
0 & 0 & 0 & 0 & 0 & -\frac{1}{\tau_{2, i}} R & C^{T}(i) \bar{P}_{i} \\
\bar{P}_{i} D(i) & \bar{P}_{i} A(i) & 0 & \bar{P}_{i} B(i) & 0 & \bar{P}_{i} C(i) & -\bar{P}_{i}
\end{array}\right)<0, \quad i \in \mathcal{S}
$$




$$
\begin{aligned}
\Xi_{i}= & \kappa_{0} Q-\Theta_{i}, \quad \kappa_{0}=(1-\underline{\pi})\left(\bar{\tau}_{1}-\underline{\tau}_{1}\right)+1 \\
\hat{\alpha}_{i}= & \tau_{2, i}+\left(1-\pi_{i i}\right)\left(\bar{\tau}_{2}-\underline{\tau}_{2}\right) \\
& +\frac{1}{2}(1-\underline{\pi})\left(\bar{\tau}_{2}-\underline{\tau}_{2}\right)\left(\bar{\tau}_{2}+\underline{\tau}_{2}-1\right) .
\end{aligned}
$$

Proof: For presentation convenience, we introduce some notations in the equation at the bottom of the page.

By Lemma 4, inequality (9) is equivalent to

$$
\hat{\Phi}_{i}+\mathcal{A}^{T}(i) \bar{P}_{i} \mathcal{A}(i)<0, \quad i \in \mathcal{S}
$$

where

$$
\hat{\Phi}_{i}=\left(\begin{array}{cccccc}
\Pi_{i} & \Omega_{i} \Lambda_{2} & \Theta_{i} \Sigma_{2} & 0 & \Delta_{i} \Upsilon_{2} & 0 \\
\Omega_{i} \Lambda_{2} & -\Omega_{i} & 0 & 0 & 0 & 0 \\
\Theta_{i} \Sigma_{2} & 0 & \Xi_{i} & 0 & 0 & 0 \\
0 & 0 & 0 & -Q & 0 & 0 \\
\Delta_{i} \Upsilon_{2} & 0 & 0 & 0 & \hat{\alpha}_{i} R-\Delta_{i} & 0 \\
0 & 0 & 0 & 0 & 0 & -\frac{1}{\tau_{2, i}} R
\end{array}\right) .
$$

Consider the stochastic Lyapunov-Krasovskii functional $V\left(\mathbf{x}_{k}, k, r(k)\right)$ as follows:

$$
\begin{aligned}
V\left(\mathbf{x}_{k}, k, r(k)\right)= & V_{1}\left(\mathbf{x}_{k}, k, r(k)\right)+V_{2}\left(\mathbf{x}_{k}, k, r(k)\right) \\
& +V_{3}\left(\mathbf{x}_{k}, k, r(k)\right)+V_{4}\left(\mathbf{x}_{k}, k, r(k)\right) \\
& +V_{5}\left(\mathbf{x}_{k}, k, r(k)\right)
\end{aligned}
$$

where

$$
\begin{aligned}
& V_{1}\left(\mathbf{x}_{k}, k, r(k)\right)=x^{T}(k) P_{r(k)} x(k) \\
& V_{2}\left(\mathbf{x}_{k}, k, r(k)\right)=\sum_{v=k-\tau_{1, r(k)}} G^{T}(x(v)) Q G(x(v)) \\
& V_{3}\left(\mathbf{x}_{k}, k, r(k)\right)=(1-\underline{\pi}) \sum_{\iota=\underline{\tau}_{1}}^{\bar{\tau}_{1}-1} \sum_{v=k-\iota}^{k-1} G^{T}(x(v)) Q G(x(v)) \\
& V_{4}\left(\mathbf{x}_{k}, k, r(k)\right)=\sum_{\iota=1}^{\tau_{2, r(k)}} \sum_{v=k-\iota}^{k-1} H^{T}(x(v)) R H(x(v)) \\
& V_{5}\left(\mathbf{x}_{k}, k, r(k)\right)=(1-\underline{\pi}) \sum_{s=\underline{\tau}_{2}+1} \sum_{\iota=1}^{\bar{\tau}_{2}} \sum_{v=k-\iota}^{k-1} H^{T}(x(v)) R H(x(v)) .
\end{aligned}
$$

Notice that $V_{2}\left(\mathbf{x}_{k}, k, r(k)\right)$ and $V_{3}\left(\mathbf{x}_{k}, k, r(k)\right)$ are used to deal with the term involving the discrete delay in (1a) while $V_{4}\left(\mathbf{x}_{k}, k, r(k)\right)$ and $V_{5}\left(\mathbf{x}_{k}, k, r(k)\right)$ are used to deal with the term involving the distributed delay.

For $i \in \mathcal{S}$, we conduct the following computation:

$$
\begin{aligned}
& \mathbb{E}\left[V_{1}\left(\mathbf{x}_{k+1}, k+1, r(k+1)\right) \mid \mathbf{x}_{k}, r(k)=i\right]-V_{1}\left(\mathbf{x}_{k}, k, i\right) \\
& \quad=\sum_{j=1}^{N} \pi_{i j} x^{T}(k+1) P_{j} x(k+1)-x^{T}(k) P_{i} x(k) \\
& \quad=\left[D(i) x(k)+A(i) F(x(k))+B(i) G\left(x\left(k-\tau_{1, i}\right)\right)\right.
\end{aligned}
$$

$$
\begin{aligned}
& \left.+C(i) \sum_{v=1}^{\tau_{2, i}} H(x(k-v))\right]^{T} \\
& \times \bar{P}_{i}\left[D(i) x(k)+A(i) F(x(k))+B(i) G\left(x\left(k-\tau_{1, i}\right)\right)\right. \\
& \left.\quad+C(i) \sum_{v=1}^{\tau_{2, i}} H(x(k-v))\right]-x^{T}(k) P_{i} x(k) \\
& =\xi^{T}(k, i) \mathcal{A}^{T}(i) \bar{P}_{i} \mathcal{A}(i) \xi(k, i)-x^{T}(k) P_{i} x(k) .
\end{aligned}
$$

and

$=\sum_{j=1}^{N} \pi_{i j} \sum_{v=k-\tau_{1, j}+1}^{k} G^{T}(x(v)) Q G(x(v))$

$-\sum_{v=k-\tau_{1, i}}^{k-1} G^{T}(x(v)) Q G(x(v))$

$=\sum_{j=1}^{N} \pi_{i j} G^{T}(x(k)) Q G(x(k))$

$-G^{T}\left(x\left(k-\tau_{1, i}\right)\right) Q G\left(x\left(k-\tau_{1, i}\right)\right)$

$+\sum_{j=1}^{N} \pi_{i j} \sum_{v=k-\tau_{1, j}+1}^{k-1} G^{T}(x(v)) Q G(x(v))$

- $\sum_{v=k-\tau_{1, i}+1}^{k-1} G^{T}(x(v)) Q G(x(v))$

$=G^{T}(x(k)) Q G(x(k))-G^{T}\left(x\left(k-\tau_{1, i}\right)\right) Q G\left(x\left(k-\tau_{1, i}\right)\right)$

$+\sum_{j \neq i} \pi_{i j}\left(\sum_{v=k-\tau_{1, j}+1}^{k-1} G^{T}(x(v)) Q G(x(v))\right.$

$\left.-\sum_{v=k-\tau_{1, i}+1}^{k-1} G^{T}(x(v)) Q G(x(v))\right)$

$\leq G^{T}(x(k)) Q G(x(k))-G^{T}\left(x\left(k-\tau_{1, i}\right)\right) Q G\left(x\left(k-\tau_{1, i}\right)\right)$

$+\sum_{j \neq i} \pi_{i j}\left(\sum_{v=k-\bar{\tau}_{1}+1}^{k-1} G^{T}(x(v)) Q G(x(v))\right.$

$\left.-\sum_{v=k-\underline{\tau}_{1}+1}^{k-1} G^{T}(x(v)) Q G(x(v))\right)$

$\leq G^{T}(x(k)) Q G(x(k))-G^{T}\left(x\left(k-\tau_{1, i}\right)\right) Q G\left(x\left(k-\tau_{1, i}\right)\right)$

$+\sum_{j \neq i} \pi_{i j} \sum_{v=k-\bar{\tau}_{1}+1}^{k-\underline{\tau}_{1}} G^{T}(x(v)) Q G(x(v))$

$\leq G^{T}(x(k)) Q G(x(k))-G^{T}\left(x\left(k-\tau_{1, i}\right)\right) Q G\left(x\left(k-\tau_{1, i}\right)\right)$

$+(1-\underline{\pi}) \sum_{v=k-\bar{\tau}_{1}+1}^{k-\underline{\tau}_{1}} G^{T}(x(v)) Q G(x(v))$

$$
\begin{aligned}
\mathbf{x}_{k} & =\left[\begin{array}{lllll}
x^{T}(k), x^{T}(k-1), \ldots, x^{T}(k-\tau)
\end{array}\right]^{T} \\
\xi(k, i) & =\left[\begin{array}{llllll}
x^{T}(k) & F^{T}(x(k)) & G^{T}(x(k)) & G^{T}\left(x\left(k-\tau_{1, i}\right)\right) & H^{T}(x(k)) & \sum_{v=1}^{\tau_{2, i}} H^{T}(x(k-v))
\end{array}\right]^{T} \\
\mathcal{A}(i) & =\left[\begin{array}{llllll}
D(i) & A(i) & 0 & B(i) & 0 & C(i)
\end{array}\right]
\end{aligned}
$$


and

$$
\begin{gathered}
\mathbb{E}\left[V_{3}\left(\mathbf{x}_{k+1}, k+1, r(k+1)\right) \mid \mathbf{x}_{k}, r(k)=i\right]-V_{3}\left(\mathbf{x}_{k}, k, i\right) \\
=(1-\underline{\pi})\left(\sum_{\iota=\underline{\tau}_{1}}^{\bar{\tau}_{1}-1} \sum_{v=k-\iota+1}^{k} G^{T}(x(v)) Q G(x(v))\right. \\
\left.\quad-\sum_{\iota=\underline{\tau}_{1}}^{\bar{\tau}_{1}-1} \sum_{v=k-\iota}^{k-1} G^{T}(x(v)) Q G(x(v))\right) \\
=(1-\underline{\pi}) \sum_{\iota=\underline{\tau}_{1}}^{\bar{\tau}_{1}-1}\left(G^{T}(x(k)) Q G(x(k))\right. \\
=(1-\underline{\pi})\left(\bar{\tau}_{1}-\underline{\tau}_{1}\right) G^{T}(x(k)) Q G(x(k)) \\
-(1-\underline{\pi}) \sum_{v=k-\bar{\tau}_{1}+1}^{k-\underline{\tau}_{1}} G^{T}(x(v)) Q G(x(v))
\end{gathered}
$$

and

$$
\mathbb{E}\left[V_{4}\left(\mathbf{x}_{k+1}, k+1, r(k+1)\right) \mid \mathbf{x}_{k}, r(k)=i\right]-V_{4}\left(\mathbf{x}_{k}, k, i\right)
$$$$
=\sum_{j=1}^{N} \pi_{i j} \sum_{\iota=1}^{\tau_{2, j}} \sum_{v=k-\iota+1}^{k} H^{T}(x(v)) R H(x(v))
$$$$
-\sum_{\iota=1}^{\tau_{2, i}} \sum_{v=k-\iota}^{k-1} H^{T}(x(v)) R H(x(v))
$$$$
=\pi_{i i}\left(\sum_{\iota=1}^{\tau_{2, i}} \sum_{v=k-\iota+1}^{k} H^{T}(x(v)) R H(x(v))\right.
$$$$
\left.-\sum_{\iota=1}^{\tau_{2, i}} \sum_{v=k-\iota}^{k-1} H^{T}(x(v)) R H(x(v))\right)
$$$$
+\sum_{j \neq i} \pi_{i j}\left(\sum_{\iota=1}^{\tau_{2, j}} \sum_{v=k-\iota+1}^{k} H^{T}(x(v)) R H(x(v))\right.
$$$$
\left.-\sum_{\iota=1}^{\tau_{2, i}} \sum_{v=k-\iota}^{k-1} H^{T}(x(v)) R H(x(v))\right)
$$$$
=\pi_{i i}\left(\sum_{\iota=1}^{\tau_{2, i}} \sum_{v=k-\iota+1}^{k} H^{T}(x(v)) R H(x(v))\right.
$$$$
\left.-\sum_{\iota=1}^{\tau_{2, i}} \sum_{v=k-\iota}^{k-1} H^{T}(x(v)) R H(x(v))\right)
$$$$
+\sum_{j \neq i} \pi_{i j}\left(\sum_{\iota=1}^{\tau_{2, i}} \sum_{v=k-\iota+1}^{k} H^{T}(x(v)) R H(x(v))\right.
$$$$
\left.-\sum_{\iota=1}^{\tau_{2, i}} \sum_{v=k-\iota}^{k-1} H^{T}(x(v)) R H(x(v))\right)
$$$$
+\sum_{j \neq i} \pi_{i j}\left(\sum_{\iota=1}^{\tau_{2, j}} \sum_{v=k-\iota+1}^{k} H^{T}(x(v)) R H(x(v))\right.
$$$$
\left.-\sum_{\iota=1}^{\tau_{2, i}} \sum_{v=k-\iota+1}^{k} H^{T}(x(v)) R H(x(v))\right)
$$

$\leq \sum_{\iota=1}^{\tau_{2, i}}\left(H^{T}(x(k)) R H(x(k))-H^{T}(x(k-\iota)) R H(x(k-\iota))\right)$

$$
\begin{aligned}
& +\sum_{j \neq i} \pi_{i j}\left(\sum_{\iota=\underline{\tau}_{2}+1}^{\bar{\tau}_{2}} \sum_{v=k-\iota+1}^{k} H^{T}(x(v)) R H(x(v))\right) \\
= & \tau_{2, i} H^{T}(x(k)) R H(x(k))-\sum_{\iota=1}^{\tau_{2, i}} H^{T}(x(k-\iota)) R H(x(k-\iota)) \\
& +\left(1-\pi_{i i}\right) \sum_{\iota=\underline{\tau}_{2}+1}^{\bar{\tau}_{2}} \sum_{v=k-\iota+1}^{k} H^{T}(x(v)) R H(x(v)) \\
\leq & \left(\tau_{2, i}+\left(1-\pi_{i i}\right)\left(\bar{\tau}_{2}-\underline{\tau}_{2}\right)\right) H^{T}(x(k)) R H(x(k)) \\
& -\sum_{v=1}^{\tau_{2, i}} H^{T}(x(k-v)) R H(x(k-v)) \\
& +(1-\underline{\pi}) \sum_{\iota=\underline{\tau}_{2}+1} \sum_{v=k-\iota+1}^{k-1} H^{T}(x(v)) R H(x(v))
\end{aligned}
$$

and

$$
\begin{array}{r}
\mathbb{E}\left[V_{5}\left(\mathbf{x}_{k+1}, k+1, r(k+1)\right) \mid \mathbf{x}_{k}, r(k)=i\right]-V_{5}\left(\mathbf{x}_{k}, k, i\right) \\
=(1-\underline{\pi})\left[\sum_{s=\underline{\tau}_{2}+1}^{\bar{\tau}_{2}} \sum_{\iota=1}^{s-1} \sum_{v=k-\iota+1}^{k} H^{T}(x(v)) R H(x(v))\right. \\
\left.-\sum_{s=\tau_{2}+1}^{\bar{\tau}_{2}} \sum_{\iota=1}^{s-1} \sum_{v=k-\iota}^{k-1} H^{T}(x(v)) R H(x(v))\right] \\
=(1-\underline{\pi})\left[\sum _ { s = \underline { \tau } _ { 2 } + 1 } ^ { \overline { \tau } _ { 2 } } \sum _ { \iota = 1 } ^ { s - 1 } \left(H^{T}(x(k)) R H(x(k))\right.\right. \\
=(1-\underline{\pi})\left[\frac{1}{2}\left(\bar{\tau}_{2}-\underline{\tau}_{2}\right)\left(\bar{\tau}_{2}+\underline{\tau}_{2}-1\right) H^{T}(x(k)) R H(x(k))\right. \\
\left.-\sum_{\iota=\underline{\tau}_{2}+1}^{\bar{\tau}_{2}} \sum_{v=k-\iota+1}^{k-1} H^{T}(x(v)) R H(x(v))\right] .(25)
\end{array}
$$

From (21)-(25), it follows that

$$
\begin{aligned}
\mathbb{E}[ & \left.V\left(\mathbf{x}_{k+1}, k+1, r(k+1)\right) \mid \mathbf{x}_{k}, r(k)=i\right]-V\left(\mathbf{x}_{k}, k, i\right) \\
\leq & \xi^{T}(k, i) \mathcal{A}^{T}(i) \bar{P}_{i} \mathcal{A}(i) \xi(k, i)-x^{T}(k) P_{i} x(k) \\
& +\kappa_{0} G^{T}(x(k)) Q G(x(k)) \\
& -G^{T}\left(x\left(k-\tau_{1, i}\right)\right) Q G\left(x\left(k-\tau_{1, i}\right)\right) \\
& +\hat{\alpha}_{i} H^{T}(x(k)) R H(x(k)) \\
& -\sum_{v=1}^{\tau_{2, i}} H^{T}(x(k-v)) R H(x(k-v))
\end{aligned}
$$

where $\kappa_{0}$ and $\hat{\alpha}_{i}$ are defined in (12) and (13), respectively.

Next we will make estimate on the terms in (26) to obtain the stability of the system (1) under given conditions.

From Lemma 2, one has

$$
\begin{aligned}
& -\sum_{v=1}^{\tau_{2, i}} H^{T}(x(k-v)) R H(x(k-v)) \\
& \quad \leq-\frac{1}{\tau_{2, i}}\left(\sum_{v=1}^{\tau_{2, i}} H(x(k-v))\right)^{T} R \sum_{v=1}^{\tau_{2, i}} H(x(k-v)) .
\end{aligned}
$$


In view of Assumptions 1 and 2 and using Lemma 3, it follows that

$$
\begin{array}{r}
x^{T}(k) \Omega_{i} \Lambda_{1} x(k)-2 x^{T}(k) \Omega_{i} \Lambda_{2} F(x(k)) \\
+F^{T}(x(k)) \Omega_{i} F(x(k)) \leq 0 \\
x^{T}(k) \Theta_{i} \Sigma_{1} x(k)-2 x^{T}(k) \Theta_{i} \Sigma_{2} G(x(k)) \\
+G^{T}(x(k)) \Theta_{i} G(x(k)) \leq 0 \\
x^{T}(k) \Delta_{i} \Upsilon_{1} x(k)-2 x^{T}(k) \Delta_{i} \Upsilon_{2} H(x(k)) \\
+H^{T}(x(k)) \Delta_{i} H(x(k)) \leq 0 .
\end{array}
$$

Substituting (27)-(30) into (26) leads to

$$
\begin{aligned}
\mathbb{E}[ & \left.V\left(\mathbf{x}_{k+1}, k+1, r(k+1)\right) \mid \mathbf{x}_{k}, r(k)=i\right]-V\left(\mathbf{x}_{k}, k, i\right) \\
\leq & \xi^{T}(k, i) \mathcal{A}^{T}(i) \bar{P}_{i} \mathcal{A}(i) \xi(k, i)-x^{T}(k) P_{i} x(k) \\
& +\kappa_{0} G^{T}(x(k)) Q G(x(k)) \\
& -G^{T}\left(x\left(k-\tau_{1, i}\right)\right) Q G\left(x\left(k-\tau_{1, i}\right)\right) \\
& +\hat{\alpha}_{i} H^{T}(x(k)) R H(x(k)) \\
& -\frac{1}{\tau_{2, i}}\left(\sum_{v=1}^{\tau_{2, i}} H(x(k-v))\right)^{T} R \sum_{v=1}^{\tau_{2, i}} H(x(k-v)) \\
- & \left(x^{T}(k) \Omega_{i} \Lambda_{1} x(k)-2 x^{T}(k) \Omega_{i} \Lambda_{2} F(x(k))\right. \\
& \left.\quad+F^{T}(x(k)) \Omega_{i} F(x(k))\right) \\
& -\left(x^{T}(k) \Theta_{i} \Sigma_{1} x(k)-2 x^{T}(k) \Theta_{i} \Sigma_{2} G(x(k))\right. \\
& \left.+G^{T}(x(k)) \Theta_{i} G(x(k))\right) \\
& -\left(x^{T}(k) \Delta_{i} \Upsilon_{1} x(k)-2 x^{T}(k) \Delta_{i} \Upsilon_{2} H(x(k))\right. \\
& \left.+H^{T}(x(k)) \Delta_{i} H(x(k))\right) \\
= & \xi^{T}(k, i)\left(\mathcal{A}^{T}(i) \bar{P}_{i} \mathcal{A}(i)+\hat{\Phi}_{i}\right) \xi(k, i) .
\end{aligned}
$$

Let $\alpha_{0}=\max _{i \in \mathcal{S}}\left\{\lambda_{\max }\left(\mathcal{A}^{T}(i) \bar{P}_{i} \mathcal{A}(i)+\hat{\Phi}_{i}\right)\right\}$. In terms of (14), it is obvious that $\alpha_{0}<0$. Therefore, from (31), it follows readily that

$$
\begin{array}{r}
\mathbb{E}\left[V\left(\mathbf{x}_{k+1}, k+1, r(k+1)\right) \mid \mathbf{x}_{k}, r(k)=i\right] \\
-V\left(\mathbf{x}_{k}, k, i\right) \leq \alpha_{0}|x(k)|^{2}
\end{array}
$$

which implies

$\mathbb{E}\left[V\left(\mathbf{x}_{k+1}, k+1, r(k+1)\right)\right]-\mathbb{E}\left[V\left(\mathbf{x}_{k}, k, r(k)\right)\right] \leq \alpha_{0} \mathbb{E}\left[|x(k)|^{2}\right]$.

For an arbitrary positive number $m$, it can be inferred from (32) that

$$
\begin{aligned}
& \mathbb{E}\left[V\left(\mathbf{x}_{m+1}, m+1, r(m+1)\right)\right]-\mathbb{E}\left[V\left(\mathbf{x}_{0}, 0, r(0)\right)\right] \\
& \leq \alpha_{0} \sum_{k=0}^{m} \mathbb{E}\left[|x(k)|^{2}\right]
\end{aligned}
$$

which results in

$$
\sum_{k=0}^{m} \mathbb{E}\left[|x(k)|^{2}\right] \leq-\frac{1}{\alpha_{0}} \mathbb{E}\left[V\left(\mathbf{x}_{0}, 0, r(0)\right)\right] .
$$

It can now be concluded that the series $\sum_{k=0}^{+\infty} \mathbb{E}\left[|x(k)|^{2}\right]$ is convergent, and therefore

$$
\lim _{k \rightarrow+\infty} \mathbb{E}\left[|x(k)|^{2}\right]=0 .
$$

This completes the proof of the theorem.
Remark 3: In Theorem 1, we have obtained the LMI-based conditions ensuring system (1) to be asymptotically stable in the mean square. The conditions are easy to be checked as LMIs can be effectively solved by the algorithms such as the interior-point method from Matlab toolbox. We like to point out that, under the same conditions, the mean square exponential stability can also be guaranteed following a similar method used in [22].

\section{SYNCHRONIZATION}

In the previous section, we have analyzed the stability for a single neural network. In this section, we will consider an array of coupled identical neural networks and deal with the synchronization problem of the coupled neural networks.

Consider a coupled system of $n_{0}$ identical neural networks described by

$$
\begin{aligned}
y_{m}(k+1)= & D(r(k)) y_{m}(k)+A(r(k)) F\left(y_{m}(k)\right) \\
& +B(r(k)) G\left(y_{m}\left(k-\tau_{1, r(k)}\right)\right) \\
& +C(r(k)) \sum_{v=1}^{\tau_{2, r(k)}} H\left(y_{m}(k-v)\right) \\
& +\sum_{\alpha=1}^{n_{0}} w_{m \alpha} \Gamma_{r(k)} y_{\alpha}(k), \quad m=1,2, \ldots, n_{0}
\end{aligned}
$$

where $y_{m}(k)=\left(y_{m 1}(k), y_{m 2}(k), \ldots, y_{m n}(k)\right)^{T}$ is the state vector of the $m$ th neural network; if $r(k)=i$, $\Gamma_{r(k)}=\operatorname{diag}\left\{\gamma_{1 i}, \gamma_{2 i}, \ldots, \gamma_{n i}\right\} \geq 0$ is a matrix linking the $j$ th state variable if $\gamma_{j i} \neq 0(j \in\{1,2, \ldots, n\})$; and $W=\left(w_{m \alpha}\right) \in \mathbb{R}^{n_{0} \times n_{0}}$ is the coupled configuration matrix of the network with $w_{m \alpha} \geq 0(m \neq \alpha)$ but not all zero. As usual, the coupling configuration matrix $W=\left(w_{m \alpha}\right)$ is symmetric (i.e., $W=W^{T}$ ) and satisfies

and

$$
\sum_{\alpha=1}^{n_{0}} w_{m \alpha}=0, \quad m=1,2, \ldots, n_{0}
$$

$$
\sum_{m=1}^{n_{0}} w_{m \alpha}=0, \quad \alpha=1,2, \ldots, n_{0} .
$$

All other symbols in (33) are defined as in the previous section.

Remark 4: The coupling satisfying $\sum_{\alpha=1}^{n_{0}} w_{m \alpha}=0$ for $1 \leq \alpha \leq n_{0}$ is called linear coupling, which is used extensively. In the case of the linear coupling, when the states of the coupled neural networks are identical to each other, the coupled networks are decoupled.

Let

$$
\begin{aligned}
y(k) & =\left(y_{1}^{T}(k), y_{2}^{T}(k), \ldots, y_{n_{0}}^{T}(k)\right)^{T} \\
\hat{F}(y(k)) & =\left(F^{T}\left(y_{1}(k)\right), F^{T}\left(y_{2}(k)\right), \ldots, F^{T}\left(y_{n_{0}}(k)\right)\right)^{T} \\
\hat{G}(y(k)) & =\left(G^{T}\left(y_{1}(k)\right), G^{T}\left(y_{2}(k)\right), \ldots, G^{T}\left(y_{n_{0}}(k)\right)\right)^{T} \\
\hat{H}(y(k)) & =\left(H^{T}\left(y_{1}(k)\right), H^{T}\left(y_{2}(k)\right), \ldots, H^{T}\left(y_{n_{0}}(k)\right)\right)^{T} .
\end{aligned}
$$

With the matrix Kronecker product, we can rewrite the coupled neural networks (33) in the following compact form:

$$
\begin{aligned}
y(k+1)= & (I \otimes D(r(k))) y(k)+(I \otimes A(r(k))) \hat{F}(y(k)) \\
& +(I \otimes B(r(k))) \hat{G}\left(y\left(k-\tau_{1, r(k)}\right)\right)+(I \otimes C(r(k))) \\
& \times \sum_{v=1}^{\tau_{2, r(k)}} \hat{H}(y(k-v))+\left(W \otimes \Gamma_{r(k)}\right) y(k) .
\end{aligned}
$$


Remark 5: It should be pointed out that the discrete and/or the distributed delay state coupling(s) can be considered in the coupled system (33) or (35) without any difficulty. However, to avoid tedious calculation and without loss of generality, we only discuss the state coupling in (33) or (35).

Definition 2: The coupled system (35) is said to be asymptotically synchronized in the mean square if the following holds: $\left.\lim _{k \rightarrow+\infty} \mathbb{E} \| y_{m}(k)-\left.y_{\alpha}(k)\right|^{2}\right]=0, \quad 1 \leq m<\alpha \leq n_{0}$.

The coupled system (35) is said to be exponentially synchronized in the mean square if, for any solution $\{y(k)\}$ of (35), there exist scalar constants $c>0$ and $0<\gamma<1$ such that

$\mathbb{E}\left[\left|y_{m}(k)-y_{\alpha}(k)\right|^{2}\right] \leq c \gamma^{k}, \quad 1 \leq m<\alpha \leq n_{0} \quad \forall k>0$.

In the sequel, for notation simplicity, we denote by $w_{i j}^{(2)}$ the $(i, j)$ entry of the matrix $W^{2}$.

Theorem 2: Under Assumptions 1 and 2, the coupled system (35) is asymptotically synchronized in the mean square if there exist a set of matrices $P_{i}>0$, two matrices $Q>0$ and $R>0$, and three sets of diagonal matrices $\Omega_{m, \alpha, i}>0, \Theta_{m, \alpha, i}>0$, and $\Delta_{m, \alpha, i}>0$ such that the following LMIs hold:

$$
\Psi_{m, \alpha, i}<0, \quad 1 \leq m<\alpha \leq n_{0}, \quad i \in \mathcal{S}
$$

where $\Psi_{m, \alpha, i}$ is defined in (37) at the bottom of the page, and $\kappa_{0}, \hat{\alpha}_{i}$, and $\bar{P}_{i}$ are defined as in Theorem 1.

Proof: For presentation convenience, we denote $\mathbf{y}_{k}=\left(y^{T}(k), y^{T}(k-1), \ldots, y^{T}(k-\tau)\right)^{T}, \mathbf{y}_{m \alpha}(k)=$ $y_{m}(k)-y_{\alpha}(k), \mathbf{f}_{m \alpha}(k)=F\left(y_{m}(k)\right)-F\left(y_{\alpha}(k)\right), \mathbf{g}_{m \alpha}(k)=$ $G\left(y_{m}(k)\right)-G\left(y_{\alpha}(k)\right), \mathbf{h}_{m \alpha}(k)=H\left(y_{m}(k)\right)-H\left(y_{\alpha}(k)\right)$, and $\widehat{\mathbf{h}}_{m \alpha}(k, r(k))=\sum_{v=1}^{\tau_{2, r(k)}} H\left(y_{m}(k-v)\right)-\sum_{v=1}^{\tau_{2}, r(k)} H\left(y_{\alpha}(k-v)\right)$.

Consider the stochastic Lyapunov-Krasovskii functional $\hat{V}\left(\mathbf{y}_{k}, k, r(k)\right)$ as follows:

$$
\begin{aligned}
& \hat{V}\left(\mathbf{y}_{k}, k, r(k)\right)=\hat{V}_{1}\left(\mathbf{y}_{k}, k, r(k)\right)+\hat{V}_{2}\left(\mathbf{y}_{k}, k, r(k)\right) \\
& \quad+\hat{V}_{3}\left(\mathbf{y}_{k}, k, r(k)\right)+\hat{V}_{4}\left(\mathbf{y}_{k}, k, r(k)\right)+\hat{V}_{5}\left(\mathbf{y}_{k}, k, r(k)\right)
\end{aligned}
$$

where

$\hat{V}_{1}\left(\mathbf{y}_{k}, k, r(k)\right)=y^{T}(k)\left(U \otimes P_{r(k)}\right) y(k)$

$\hat{V}_{2}\left(\mathbf{y}_{k}, k, r(k)\right)=\sum_{v=k-\tau_{1, r(k)}}^{k-1} \hat{G}^{T}(y(v))(U \otimes Q) \hat{G}(y(v))$
$\hat{V}_{3}\left(\mathbf{y}_{k}, k, r(k)\right)=(1-\underline{\pi}) \sum_{\iota=\underline{\tau}_{1}}^{\bar{\tau}_{1}-1} \sum_{v=k-\iota}^{k-1} \hat{G}^{T}(y(v))(U \otimes Q) \hat{G}(y(v))$

$\hat{V}_{4}\left(\mathbf{y}_{k}, k, r(k)\right)=\sum_{\iota=1}^{\tau_{2, r(k)}} \sum_{v=k-\iota}^{k-1} \hat{H}^{T}(y(v))(U \otimes R) \hat{H}(y(v))$

$\hat{V}_{5}\left(\mathbf{y}_{k}, k, r(k)\right)=(1-\underline{\pi})$

$$
\times \sum_{s=\underline{\tau}_{2}+1}^{\bar{\tau}_{2}} \sum_{\iota=1}^{s-1} \sum_{v=k-\iota}^{k-1} \hat{H}^{T}(y(v))(U \otimes R) \hat{H}(y(v))
$$

with

$$
U=\left(\begin{array}{cccc}
n_{0}-1 & -1 & \cdots & -1 \\
-1 & n_{0}-1 & \cdots & -1 \\
\cdots & \cdots & \cdots & \cdots \\
-1 & -1 & \cdots & n_{0}-1
\end{array}\right)_{n_{0} \times n_{0}}
$$

For $i \in \mathcal{S}$, we perform the following computation:

$$
\begin{gathered}
\mathbb{E}\left[\hat{V}_{1}\left(\mathbf{y}_{k+1}, k+1, r(k+1)\right) \mid \mathbf{y}_{k}, r(k)=i\right]-\hat{V}_{1}\left(\mathbf{y}_{k}, k, i\right) \\
=\sum_{j=1}^{N} \pi_{i j} y^{T}(k+1)\left(U \otimes P_{j}\right) y(k+1)-y^{T}(k)\left(U \otimes P_{i}\right) y(k) \\
=[(I \otimes D(i)) y(k)+(I \otimes A(i)) \hat{F}(y(k)) \\
\quad+(I \otimes B(i)) \hat{G}\left(y\left(k-\tau_{1, i}\right)\right) \\
\left.\quad+(I \otimes C(i)) \sum_{v=1}^{\tau_{2, i}} \hat{H}(y(k-v))+\left(W \otimes \Gamma_{i}\right) y(k)\right]^{T} \\
\times\left(U \otimes \bar{P}_{i}\right)[(I \otimes D(i)) y(k)+(I \otimes A(i)) \hat{F}(y(k)) \\
\quad+(I \otimes B(i)) \hat{G}\left(y\left(k-\tau_{1, i}\right)\right) \\
+(I \otimes C(i)) \sum_{v=1}^{\tau_{2, i}} \hat{H}(y(k-v)) \\
\left.+\left(W \otimes \Gamma_{i}\right) y(k)\right]-y^{T}(k)\left(U \otimes P_{i}\right) y(k)
\end{gathered}
$$

$$
\begin{aligned}
& \Psi_{m, \alpha, i}=\left(\begin{array}{cccccc}
\Xi_{m, \alpha, i}^{(1,1)} & \Xi_{m, \alpha, i}^{(1,2)} & \Theta_{m, \alpha, i} \Sigma_{2} & \Xi_{m, \alpha, i}^{(1,4)} & \Delta_{m, \alpha, i} \Upsilon_{2} & \Xi_{m, \alpha, i}^{(1,6)} \\
* & \Xi_{m, \alpha, i}^{(2,2)} & 0 & A^{T}(i) \bar{P}_{i} B(i) & 0 & A^{T}(i) \bar{P}_{i} C(i) \\
* & * & \Xi_{m, \alpha, i}^{(3,3)} & 0 & 0 & 0 \\
* & * & * & \Xi_{m, \alpha, i}^{(4,4)} & 0 & B^{T}(i) \bar{P}_{i} C(i) \\
* & * & * & * & \hat{\alpha}_{i} R-\Delta_{m, \alpha, i} & 0 \\
* & * & * & * & * & \Xi_{m, \alpha, i}^{(6,6)}
\end{array}\right) \\
& \Xi_{m, \alpha, i}^{(1,1)}=-P_{i}+D(i) \bar{P}_{i} D(i)-n_{0} w_{m \alpha}^{(2)} \Gamma_{i} \bar{P}_{i} \Gamma_{i}-\left(n_{0} w_{m \alpha} D(i) \bar{P}_{i} \Gamma_{i}+n_{0} w_{m \alpha} \Gamma_{i} \bar{P}_{i} D(i)\right)-\Omega_{m, \alpha, i} \Lambda_{1}-\Theta_{m, \alpha, i} \Sigma_{1}-\Delta_{m, \alpha, i} \Upsilon_{1} \\
& \Xi_{m, \alpha, i}^{(1,2)}=D(i) \bar{P}_{i} A(i)-n_{0} w_{m \alpha} \Gamma_{i} \bar{P}_{i} A(i)+\Omega_{m, \alpha, i} \Lambda_{2}, \quad \Xi_{m, \alpha, i}^{(1,4)}=D(i) \bar{P}_{i} B(i)-n_{0} w_{m \alpha} \Gamma_{i} \bar{P}_{i} B(i) \\
& \Xi_{m, \alpha, i}^{(1,6)}=D(i) \bar{P}_{i} C(i)-n_{0} w_{m \alpha} \Gamma_{i} \bar{P}_{i} C(i), \quad \Xi_{m, \alpha, i}^{(2,2)}=A^{T}(i) \bar{P}_{i} A(i)-\Omega_{m, \alpha, i} \\
& \Xi_{m, \alpha, i}^{(3,3)}=\kappa_{0} Q-\Theta_{m, \alpha, i}, \quad \Xi_{m, \alpha, i}^{(4,4)}=B^{T}(i) \bar{P}_{i} B(i)-Q, \quad \Xi_{m, \alpha, i}^{(6,6)}=C^{T}(i) \bar{P}_{i} C(i)-\frac{1}{\tau_{2, i}} R
\end{aligned}
$$




$$
\begin{aligned}
= & y^{T}(k)(I \otimes D(i))\left(U \otimes \bar{P}_{i}\right)(I \otimes D(i)) y(k) \\
& +\hat{F}^{T}(y(k))\left(I \otimes A^{T}(i)\right)\left(U \otimes \bar{P}_{i}\right)(I \otimes A(i)) \hat{F}(y(k)) \\
& +\hat{G}^{T}\left(y\left(k-\tau_{1, i}\right)\right)\left(I \otimes B^{T}(i)\right)\left(U \otimes \bar{P}_{i}\right)(I \otimes B(i)) \\
& \times \hat{G}\left(y\left(k-\tau_{1, i}\right)\right)+\left(\sum_{v=1}^{\tau_{2, i}} \hat{H}(y(k-v))^{T}\left(I \otimes C^{T}(i)\right)\right. \\
& \times\left(U \otimes \bar{P}_{i}\right)(I \otimes C(i))\left(\sum_{v=1}^{\tau_{2, i}} \hat{H}(y(k-v))\right. \\
& +y^{T}(k)\left(W \otimes \Gamma_{i}\right)\left(U \otimes \bar{P}_{i}\right)\left(W \otimes \Gamma_{i}\right) y(k) \\
& +2 y^{T}(k)(I \otimes D(i))\left(U \otimes \bar{P}_{i}\right)(I \otimes A(i)) \hat{F}(y(k)) \\
& +2 y^{T}(k)(I \otimes D(i))\left(U \otimes \bar{P}_{i}\right)(I \otimes B(i)) \hat{G}\left(y\left(k-\tau_{1, i}\right)\right) \\
& +2 y^{T}(k)(I \otimes D(i))\left(U \otimes \bar{P}_{i}\right)(I \otimes C(i)) \\
& \times \sum_{2, i} \hat{H}(y(k-v))+2 y^{T}(k)(I \otimes D(i))\left(U \otimes \bar{P}_{i}\right) \\
& \times\left(W \otimes \Gamma_{i}\right) y(k)+2 \hat{F}^{T}(y(k))\left(I \otimes A^{T}(i)\right)\left(U \otimes \bar{P}_{i}\right) \\
& \times(I \otimes B(i)) \hat{G}\left(y\left(k-\tau_{1, i}\right)\right)+2 \hat{F}^{T}(y(k))\left(I \otimes A^{T}(i)\right) \\
& \times\left(U \otimes \bar{P}_{i}\right)(I \otimes C(i))\left(\sum_{v=1}^{\tau_{2, i}} \hat{H}(y(k-v))\right. \\
& +2 \hat{F}^{T}(y(k))\left(I \otimes A^{T}(i)\right)\left(U \otimes \bar{P}_{i}\right)\left(W \otimes \Gamma_{i}\right) y(k) \\
& +2 \hat{G}^{T}\left(y\left(k-\tau_{1, i}\right)\right)\left(I \otimes B^{T}(i)\right)\left(U \otimes \bar{P}_{i}\right) \\
& \times(I \otimes C(i))\left(\sum_{v=1}^{\tau_{2, i}} \hat{H}(y(k-v))\right. \\
& +2 \hat{G}^{T}\left(y\left(k-\tau_{1, i}\right)\right)\left(I \otimes B^{T}(i)\right)\left(U \otimes \bar{P}_{i}\right)\left(W \otimes \Gamma_{i}\right) y(k) \\
& +\left(\sum_{v=1} \hat{H}_{2, i}(y(k-v))\left(I \otimes C^{T}(i)\right)\left(U \otimes \bar{P}_{i}\right)\right. \\
& (k)-y^{T}(k)\left(U \otimes P_{i}\right) y(k) . \\
&
\end{aligned}
$$

Notice that

$$
\begin{aligned}
\left(W \otimes \Gamma_{i}\right)\left(U \otimes \bar{P}_{i}\right)\left(W \otimes \Gamma_{i}\right) & =(W U W) \otimes\left(\Gamma_{i} \bar{P}_{i} \Gamma_{i}\right) \\
& =n_{0} W^{2} \otimes\left(\Gamma_{i} \bar{P}_{i} \Gamma_{i}\right) \\
(I \otimes D(i))\left(U \otimes \bar{P}_{i}\right)\left(W \otimes \Gamma_{i}\right) & =(U W) \otimes\left(D(i) \bar{P}_{i} \Gamma_{i}\right) \\
& =n_{0} W \otimes\left(D(i) \bar{P}_{i} \Gamma_{i}\right) \\
\left(I \otimes A^{T}(i)\right)\left(U \otimes \bar{P}_{i}\right)\left(W \otimes \Gamma_{i}\right) & =(U W) \otimes\left(A^{T}(i) \bar{P}_{i} \Gamma_{i}\right) \\
& =n_{0} W \otimes\left(A^{T}(i) \bar{P}_{i} \Gamma_{i}\right) \\
\left(I \otimes B^{T}(i)\right)\left(U \otimes \bar{P}_{i}\right)\left(W \otimes \Gamma_{i}\right) & =(U W) \otimes\left(B^{T}(i) \bar{P}_{i} \Gamma_{i}\right) \\
& =n_{0} W \otimes\left(B^{T}(i) \bar{P}_{i} \Gamma_{i}\right) \\
\left(I \otimes C^{T}(i)\right)\left(U \otimes \bar{P}_{i}\right)\left(W \otimes \Gamma_{i}\right) & =(U W) \otimes\left(C^{T}(i) \bar{P}_{i} \Gamma_{i}\right) \\
& =n_{0} W \otimes\left(C^{T}(i) \bar{P}_{i} \Gamma_{i}\right) .
\end{aligned}
$$

Substituting (45)-(49) into (44) leads to

$$
\begin{aligned}
& \mathbb{E}\left[\hat{V}_{1}\left(\mathbf{y}_{k+1}, k+1, r(k+1)\right) \mid \mathbf{y}_{k}, r(k)=i\right]-\hat{V}_{1}\left(\mathbf{y}_{k}, k, i\right) \\
& =y^{T}(k)\left(U \otimes\left(D(i) \bar{P}_{i} D(i)\right)\right) y(k) \\
& \quad+\hat{F}^{T}(y(k))\left(U \otimes\left(A^{T}(i) \bar{P}_{i} A(i)\right)\right) \hat{F}(y(k)) \\
& \quad+\hat{G}^{T}\left(y\left(k-\tau_{1, i}\right)\right)\left(U \otimes\left(B^{T}(i) \bar{P}_{i} B(i)\right)\right) \hat{G}\left(y\left(k-\tau_{1, i}\right)\right) \\
& \quad+\left(\sum_{v=1}^{\tau_{2, i}} \hat{H}(y(k-v))^{T}\left(U \otimes\left(C^{T}(i) \bar{P}_{i} C(i)\right)\right)\right.
\end{aligned}
$$

$$
\begin{aligned}
& \times\left(\sum_{v=1}^{\tau_{2, i}} \hat{H}(y(k-v))+y^{T}(k)\left(n_{0} W^{2} \otimes\left(\Gamma_{i} \bar{P}_{i} \Gamma_{i}\right)\right) y(k)\right. \\
& +2 y^{T}(k)\left(U \otimes\left(D(i) \bar{P}_{i} A(i)\right)\right) \hat{F}(y(k)) \\
& +2 y^{T}(k)\left(U \otimes\left(D(i) \bar{P}_{i} B(i)\right)\right) \hat{G}\left(y\left(k-\tau_{1, i}\right)\right) \\
& +2 y^{T}(k)\left(U \otimes\left(D(i) \bar{P}_{i} C(i)\right)\right) \sum_{v=1}^{\tau_{2, i}} \hat{H}(y(k-v)) \\
& +2 y^{T}(k)\left(n_{0} W \otimes\left(D(i) \bar{P}_{i} \Gamma_{i}\right)\right) y(k) \\
& +2 \hat{F}^{T}(y(k))\left(U \otimes\left(A^{T}(i) \bar{P}_{i} B(i)\right)\right) \hat{G}\left(y\left(k-\tau_{1, i}\right)\right) \\
& +2 \hat{F}^{T}(y(k))\left(U \otimes\left(A^{T}(i) \bar{P}_{i} C(i)\right)\right)\left(\sum_{v=1}^{\tau_{2, i}} \hat{H}(y(k-v))\right. \\
& +2 \hat{F}^{T}(y(k))\left(n_{0} W \otimes\left(A^{T}(i) \bar{P}_{i} \Gamma_{i}\right)\right) y(k) \\
& +2 \hat{G}^{T}\left(y\left(k-\tau_{1, i}\right)\right)\left(U \otimes\left(B^{T}(i) \bar{P}_{i} C(i)\right)\right) \\
& \times\left(\sum_{v=1}^{\tau_{2, i}} \hat{H}(y(k-v))+2 \hat{G}^{T}\left(y\left(k-\tau_{1, i}\right)\right)\right. \\
& \times\left(n_{0} W \otimes\left(B^{T}(i) \bar{P}_{i} \Gamma_{i}\right)\right) y(k)+2\left(\sum_{v=1}^{\tau_{2, i}} \hat{H}(y(k-v))^{T}\right. \\
& \times\left(n_{0} W \otimes\left(C^{T}(i) \bar{P}_{i} \Gamma_{i}\right)\right) y(k)-y^{T}(k)\left(U \otimes P_{i}\right) y(k) .(50)
\end{aligned}
$$

Similar to the proof in previous section, we have

$$
\begin{aligned}
& \mathbb{E}\left[\hat{V}_{2}\left(\mathbf{y}_{k+1}, k+1, r(k+1)\right) \mid \mathbf{y}_{k}, r(k)=i\right]-\hat{V}_{2}\left(\mathbf{y}_{k}, k, i\right) \\
& \leq \hat{G}^{T}(y(k))(U \otimes Q) \hat{G}(y(k)) \\
& \quad-\hat{G}^{T}\left(y\left(k-\tau_{1, i}\right)\right)(U \otimes Q) \hat{G}\left(y\left(k-\tau_{1, i}\right)\right) \\
& \quad+(1-\underline{\pi}) \sum_{v=k-\bar{\tau}_{1}+1}^{k-\underline{\tau}_{1}} \hat{G}^{T}(y(v))(U \otimes Q) \hat{G}(y(v))
\end{aligned}
$$

$\mathbb{E}\left[\hat{V}_{3}\left(\mathbf{y}_{k+1}, k+1, r(k+1)\right) \mid \mathbf{y}_{k}, r(k)=i\right]-\hat{V}_{3}\left(\mathbf{y}_{k}, k, i\right)$

$=(1-\underline{\pi})\left(\bar{\tau}_{1}-\underline{\tau}_{1}\right) \hat{G}^{T}(y(k))(U \otimes Q) \hat{G}(y(k))$

$$
-(1-\underline{\pi}) \sum_{v=k-\bar{\tau}_{1}+1}^{k-\tau_{1}} \hat{G}^{T}(y(v))(U \otimes Q) \hat{G}(y(v))
$$

$\mathbb{E}\left[\hat{V}_{4}\left(\mathbf{y}_{k+1}, k+1, r(k+1)\right) \mid \mathbf{y}_{k}, r(k)=i\right]-\hat{V}_{4}\left(\mathbf{y}_{k}, k, i\right)$ $\leq\left(\tau_{2, i}+\left(1-\pi_{i i}\right)\left(\bar{\tau}_{2}-\underline{\tau}_{2}\right)\right) \hat{H}^{T}(y(k))(U \otimes R) \hat{H}(y(k))$

$$
\begin{aligned}
& -\sum_{v=1}^{\tau_{2, i}} \hat{H}^{T}(y(k-v))(U \otimes R) \hat{H}(y(k-v)) \\
& +(1-\underline{\pi}) \sum_{\iota=\underline{\tau}_{2}+1}^{\bar{\tau}_{2}} \sum_{v=k-\iota+1}^{k-1} \hat{H}^{T}(y(v))(U \otimes R) \hat{H}(y(v))
\end{aligned}
$$

$$
\begin{gathered}
\mathbb{E}\left[\hat{V}_{5}\left(\mathbf{y}_{k+1}, k+1, r(k+1)\right) \mid \mathbf{y}_{k}, r(k)=i\right]-\hat{V}_{5}\left(\mathbf{y}_{k}, k, i\right) \\
=(1-\underline{\pi})\left[\frac{1}{2}\left(\bar{\tau}_{2}-\underline{\tau}_{2}\right)\left(\bar{\tau}_{2}+\underline{\tau}_{2}-1\right) \hat{H}^{T}(y(k))(U \otimes R)\right. \\
\quad \times \hat{H}(y(k))-\sum_{\iota=\underline{\tau}_{2}+1}^{\bar{\tau}_{2}} \sum_{v=k-\imath+1}^{k-1} \hat{H}^{T}(y(v)) \\
\quad \times(U \otimes R) \hat{H}(y(v))] .
\end{gathered}
$$


Therefore, it follows that

$$
\begin{aligned}
& \mathbb{E}\left[\hat{V}\left(\mathbf{y}_{k+1}, k+1, r(k+1)\right) \mid \mathbf{y}_{k}, r(k)=i\right]-\hat{V}\left(\mathbf{y}_{k}, k, i\right) \\
& \leq y^{T}(k)\left(U \otimes\left(D(i) \bar{P}_{i} D(i)\right)\right) y(k) \\
& +\hat{F}^{T}(y(k))\left(U \otimes\left(A^{T}(i) \bar{P}_{i} A(i)\right)\right) \hat{F}(y(k)) \\
& +\hat{G}^{T}\left(y\left(k-\tau_{1, i}\right)\right)\left(U \otimes\left(B^{T}(i) \bar{P}_{i} B(i)\right)\right) \\
& \times \hat{G}\left(y\left(k-\tau_{1}(k)\right)\right)+\left(\sum_{v=1}^{\tau_{2, i}} \hat{H}(y(k-v))^{T}\right. \\
& \times\left(U \otimes\left(C^{T}(i) \bar{P}_{i} C(i)\right)\right)\left(\sum_{v=1}^{\tau_{2, i}} \hat{H}(y(k-v))\right. \\
& +y^{T}(k)\left(n_{0} W^{2} \otimes\left(\Gamma_{i} \bar{P}_{i} \Gamma_{i}\right)\right) y(k) \\
& +2 y^{T}(k)\left(U \otimes\left(D(i) \bar{P}_{i} A(i)\right)\right) \hat{F}(y(k)) \\
& +2 y^{T}(k)\left(U \otimes\left(D(i) \bar{P}_{i} B(i)\right)\right) \hat{G}\left(y\left(k-\tau_{1, i}\right)\right) \\
& +2 y^{T}(k)\left(U \otimes\left(D(i) \bar{P}_{i} C(i)\right)\right) \sum_{v=1}^{\tau_{2, i}} \hat{H}(y(k-v)) \\
& +2 y^{T}(k)\left(n_{0} W \otimes\left(D(i) \bar{P}_{i} \Gamma_{i}\right)\right) y(k) \\
& +2 \hat{F}^{T}(y(k))\left(U \otimes\left(A^{T}(i) \bar{P}_{i} B(i)\right)\right) \hat{G}\left(y\left(k-\tau_{1, i}\right)\right) \\
& +2 \hat{F}^{T}(y(k))\left(U \otimes\left(A^{T}(i) \bar{P}_{i} C(i)\right)\right)\left(\sum_{v=1}^{\tau_{2, i}} \hat{H}(y(k-v))\right. \\
& +2 \hat{F}^{T}(y(k))\left(n_{0} W \otimes\left(A^{T}(i) \bar{P}_{i} \Gamma_{i}\right)\right) y(k) \\
& +2 \hat{G}^{T}\left(y\left(k-\tau_{1, i}\right)\right)\left(U \otimes\left(B^{T}(i) \bar{P}_{i} C(i)\right)\right) \\
& \times\left(\sum_{v=1}^{\tau_{2, i}} \hat{H}(y(k-v))+2 \hat{G}^{T}\left(y\left(k-\tau_{1, i}\right)\right)\right. \\
& \times\left(n_{0} W \otimes\left(B^{T}(i) \bar{P}_{i} \Gamma_{i}\right)\right) y(k) \\
& +2\left(\sum_{v=1}^{\tau_{2, i}} \hat{H}(y(k-v))^{T}\left(n_{0} W \otimes\left(C^{T}(i) \bar{P}_{i} \Gamma_{i}\right)\right) y(k)\right. \\
& -y^{T}(k)\left(U \otimes P_{i}\right) y(k)+\kappa_{0} \hat{G}^{T}(y(k))(U \otimes Q) \hat{G}(y(k)) \\
& -\hat{G}^{T}\left(y\left(k-\tau_{1, i}\right)\right)(U \otimes Q) \hat{G}\left(y\left(k-\tau_{1, i}\right)\right) \\
& +\hat{\alpha}_{i} \hat{H}^{T}(y(k-v))(U \otimes R) \hat{H}(y(k-v)) \\
& -\sum_{v=1}^{\tau_{2, i}} \hat{H}^{T}(y(k-v))(U \otimes R) \hat{H}(y(k-v)) \text {. }
\end{aligned}
$$

Using Lemmas 5 and 6, we derive from (55) that

$$
\begin{aligned}
& \mathbb{E}\left[\hat { V } \left(\mathbf{y}_{k+1}, k+1,\right.\right.\left.r(k+1)) \mid \mathbf{y}_{k}, r(k)=i\right]-\hat{V}\left(\mathbf{y}_{k}, k, i\right) \\
& \leq \sum_{1 \leq m<\alpha \leq n_{0}}\left[\mathbf { y } _ { m \alpha } ^ { T } ( k ) \left(-P_{i}+D(i) \bar{P}_{i} D(i)\right.\right. \\
& \quad-n_{0} w_{m \alpha}^{(2)} \Gamma_{i} \bar{P}_{i} \Gamma_{i} \\
&\left.\quad-2 n_{0} w_{m \alpha} D(i) \bar{P}_{i} \Gamma_{i}\right) \mathbf{y}_{m \alpha}^{T}(k) \\
& \\
&+\mathbf{f}_{m \alpha}^{T}(k) A^{T}(i) \bar{P}_{i} A(i) \mathbf{f}_{m \alpha}(k) \\
&+\kappa_{0} \mathbf{g}_{m \alpha}^{T}(k) Q \mathbf{g}_{m \alpha}(k)+\mathbf{g}_{m \alpha}^{T}\left(k-\tau_{1, i}\right) \\
& \\
& \times\left(B^{T}(i) \bar{P}_{i} B(i)-Q\right) \mathbf{g}_{m \alpha}\left(k-\tau_{1, i}\right) \\
&+\hat{\alpha}_{i} \mathbf{h}_{m \alpha}^{T}(k) R \mathbf{h}_{m \alpha}(k)+\widehat{\mathbf{h}}_{m \alpha}^{T}(k, i) C^{T}(i) \\
& \times \bar{P}_{i} C(i) \widehat{\mathbf{h}}_{m \alpha}(k, i)+2 \mathbf{y}_{m \alpha}^{T}(k) \\
& \times\left(D(i) \bar{P}_{i} A(i)-n_{0} w_{m \alpha} \Gamma_{i} \bar{P}_{i} A(i)\right) \mathbf{f}_{m \alpha}(k) \\
&+2 \mathbf{y}_{m \alpha}^{T}(k)\left(D(i) \bar{P}_{i} B(i)-n_{0} w_{m \alpha} \Gamma_{i} \bar{P}_{i} B(i)\right)
\end{aligned}
$$

$$
\begin{aligned}
& \times \mathbf{g}_{m \alpha}\left(k-\tau_{1, i}\right)+2 \mathbf{y}_{m \alpha}(k) \\
& \times\left(D(i) \bar{P}_{i} C(i)-n_{0} w_{m \alpha} \Gamma_{i} \bar{P}_{i} C(i)\right) \\
& \times \widehat{\mathbf{h}}_{m \alpha}(k, i)+2 \mathbf{f}_{m \alpha}^{T}(k) A^{T}(i) \bar{P}_{i} B(i) \\
& \times \mathbf{g}_{m \alpha}\left(k-\tau_{1, i}\right)+2 \mathbf{f}_{m \alpha}^{T}(k) A^{T}(i) \bar{P}_{i} C(i) \\
& \times \widehat{\mathbf{h}}_{m \alpha}(k, i)+2 \mathbf{g}_{m \alpha}^{T}\left(k-\tau_{1, i}\right) B^{T}(i) \bar{P}_{i} C(i) \\
& \times \widehat{\mathbf{h}}_{m \alpha}(k, i) \\
& -\sum_{v=1}^{\tau_{2, i}}\left(H\left(y_{m}(k-v)\right)-H\left(y_{\alpha}(k-v)\right)\right)^{T} \\
& \left.\times R\left(H\left(y_{m}(k-v)\right)-H\left(y_{\alpha}(k-v)\right)\right)\right] .
\end{aligned}
$$

For the same reason as in Theorem 1, we have

$$
\begin{gathered}
\mathbf{y}_{m \alpha}^{T}(k) \Omega_{m, \alpha, i} \Lambda_{1} \mathbf{y}_{m \alpha}(k)-2 \mathbf{y}_{m \alpha}^{T}(k) \Omega_{m, \alpha, i} \Lambda_{2} \mathbf{f}_{m \alpha}(k) \\
+\mathbf{f}_{m \alpha}^{T}(k) \Omega_{m, \alpha, i} \mathbf{f}_{m \alpha}(k) \leq 0 \\
\mathbf{y}_{m \alpha}^{T}(k) \Theta_{m, \alpha, i} \Sigma_{1} \mathbf{y}_{m \alpha}(k)-2 \mathbf{y}_{m \alpha}^{T}(k) \Theta_{m, \alpha, i} \Sigma_{2} \mathbf{g}_{m \alpha}(k) \\
+\mathbf{g}_{m \alpha}^{T}(k) \Theta_{m, \alpha, i} \mathbf{g}_{m \alpha}(k) \leq 0 \\
\mathbf{y}_{m \alpha}^{T}(k) \Delta_{m, \alpha, i} \Upsilon_{1} \mathbf{y}_{m \alpha}(k)-2 \mathbf{y}_{m \alpha}^{T}(k) \Delta_{m, \alpha, i} \Upsilon_{2} \mathbf{h}_{m \alpha}(k) \\
+\mathbf{h}_{m \alpha}^{T}(k) \Delta_{m, \alpha, i} \mathbf{h}_{m \alpha}(k) \leq 0
\end{gathered}
$$

and

$$
\begin{aligned}
-\sum_{v=1}^{\tau_{2, i}}\left(H\left(y_{m}(k-v)\right)-H\left(y_{\alpha}(k-v)\right)\right)^{T} \\
\times R\left(H\left(y_{m}(k-v)\right)-H\left(y_{\alpha}(k-v)\right)\right) \\
\leq-\frac{1}{\tau_{2, i}}\left(\sum_{v=1}^{\tau_{2, i}} H\left(y_{m}(k-v)\right)-H\left(y_{\alpha}(k-v)\right)\right)^{T} \\
\quad \times R\left(\sum_{v=1}^{\tau_{2, i}} H\left(y_{m}(k-v)\right)-H\left(y_{\alpha}(k-v)\right)\right) \\
=-\frac{1}{\tau_{2, i}} \widehat{\mathbf{h}}_{m \alpha}^{T}(k, i) R \widehat{\mathbf{h}}_{m \alpha}(k, i)
\end{aligned}
$$

Therefore, from (56) as well as (57)-(60), it follows that

$$
\begin{aligned}
\mathbb{E}\left[\hat { V } \left(\mathbf{y}_{k+1}, k+1,\right.\right. & \left.r(k+1)) \mid \mathbf{y}_{k}, r(k)=i\right]-\hat{V}\left(\mathbf{y}_{k}, k, i\right) \\
\leq \sum_{1 \leq m<\alpha \leq n_{0}}[ & \mathbf{y}_{m \alpha}^{T}(k)\left(-P_{i}+D(i) \bar{P}_{i} D(i)\right. \\
& -n_{0} w_{m \alpha}^{(2)} \Gamma_{i} \bar{P}_{i} \Gamma_{i} \\
& \left.-2 n_{0} w_{m \alpha} D(i) \bar{P}_{i} \Gamma_{i}\right) \mathbf{y}_{m \alpha}(k) \\
& +\mathbf{f}_{m \alpha}^{T}(k) A^{T}(i) \bar{P}_{i} A(i) \mathbf{f}_{m \alpha}(k) \\
& +\kappa_{0} \mathbf{g}_{m \alpha}^{T}(k) Q \mathbf{g}_{m \alpha}(k)+\mathbf{g}_{m \alpha}^{T}\left(k-\tau_{1, i}\right) \\
& \times\left(B^{T}(i) \bar{P}_{i} B(i)-Q\right) \mathbf{g}_{m \alpha}\left(k-\tau_{1, i}\right) \\
& +\hat{\alpha}_{i} \mathbf{h}_{m \alpha}^{T}(k) R \mathbf{h}_{m \alpha}(k)+\widehat{\mathbf{h}}_{m \alpha}^{T}(k, i) C^{T}(i) \\
& \times \bar{P}_{i} C(i) \widehat{\mathbf{h}}_{m \alpha}(k, i)+2 \mathbf{y}_{m \alpha}^{T}(k) \\
& \times\left(D(i) \bar{P}_{i} A(i)-n_{0} w_{m \alpha} \Gamma_{i} \bar{P}_{i} A(i)\right) \\
& \times \mathbf{f}_{m \alpha}(k)+2 \mathbf{y}_{m \alpha}^{T}(k) \\
& \times\left(D(i) \bar{P}_{i} B(i)-n_{0} w_{m \alpha} \Gamma_{i} \bar{P}_{i} B(i)\right) \\
& \times \mathbf{g}_{m \alpha}\left(k-\tau_{1, i}\right)+2 \mathbf{y}_{m \alpha}(k) \\
& \times\left(D(i) \bar{P}_{i} C(i)-n_{0} w_{m \alpha} \Gamma_{i} \bar{P}_{i} C(i)\right)
\end{aligned}
$$




$$
\begin{gathered}
\times \widehat{\mathbf{h}}_{m \alpha}(k, i)+2 \mathbf{f}_{m \alpha}^{T}(k) A^{T}(i) \bar{P}_{i} B(i) \\
\times \mathbf{g}_{m \alpha}\left(k-\tau_{1, i}\right)+2 \mathbf{f}_{m \alpha}^{T}(k) A^{T}(i) \bar{P}_{i} C(i) \\
\times \widehat{\mathbf{h}}_{m \alpha}(k, i)+2 \mathbf{g}_{m \alpha}^{T}\left(k-\tau_{1, i}\right) B^{T}(i) \bar{P}_{i} C(i) \\
\times \widehat{\mathbf{h}}_{m \alpha}(k, i)-\frac{1}{\tau_{2, i}} \widehat{\mathbf{h}}_{m \alpha}^{T}(k, i) R \widehat{\mathbf{h}}_{m \alpha}(k, i) \\
-\left(\mathbf{y}_{m \alpha}^{T}(k) \Omega_{m, \alpha, i} \Lambda_{1} \mathbf{y}_{m \alpha}(k)\right. \\
\quad-2 \mathbf{y}_{m \alpha}^{T}(k) \Omega_{m, \alpha, i} \Lambda_{2} \mathbf{f}_{m \alpha}(k) \\
\left.\quad+\mathbf{f}_{m \alpha}^{T}(k) \Omega_{m, \alpha, i} \mathbf{f}_{m \alpha}(k)\right) \\
-\left(\mathbf{y}_{m \alpha}^{T}(k) \Theta_{m, \alpha, i} \Sigma_{1} \mathbf{y}_{m \alpha}(k)\right. \\
\quad-2 \mathbf{y}_{m \alpha}^{T}(k) \Theta_{m, \alpha, i} \Sigma_{2} \mathbf{g}_{m \alpha}(k) \\
\left.\quad+\mathbf{g}_{m \alpha}^{T}(k) \Theta_{m, \alpha, i} \mathbf{g}_{m \alpha}(k)\right) \\
-\left(\mathbf{y}_{m \alpha}^{T}(k) \Delta_{m, \alpha, i} \Upsilon_{1} \mathbf{y}_{m \alpha}(k)\right. \\
-2 \mathbf{y}_{m \alpha}^{T}(k) \Delta_{m, \alpha, i} \Upsilon_{2} \mathbf{h}_{m \alpha}(k) \\
\left.\left.\quad+\mathbf{h}_{m \alpha}^{T}(k) \Delta_{m, \alpha, i} \mathbf{h}_{m \alpha}(k)\right)\right] \\
\hat{\xi}_{m \alpha}^{T}(k, i) \Psi_{m, \alpha, i} \hat{\xi}_{m \alpha}(k, i) \\
\sum_{1 \leq m<\alpha \leq n_{0}}
\end{gathered}
$$

where $\hat{\xi}_{m \alpha}(k, i)$ is defined in the equation at the bottom of the page.

Let $c_{0}=\max \left\{\lambda_{\max }\left(\Psi_{m, \alpha, i}\right) \mid 1 \leq m<\alpha \leq n_{0}, i \in \mathcal{S}\right\}$. Then, it is obvious that $c_{0}<0$, and $\left|\hat{\hat{\xi}}_{m \alpha}(k, i)\right|^{2} \leq\left|\mathbf{y}_{m \alpha}(k)\right|^{2}$. From (61), it follows that

$$
\begin{aligned}
\mathbb{E}\left[\hat{V}\left(\mathbf{y}_{k+1}, k+1, r(k+1)\right) \mid \mathbf{y}_{k}, r(k)\right. & =i]-\hat{V}\left(\mathbf{y}_{k}, k, i\right) \\
\leq & \sum_{1 \leq m<\alpha \leq n_{0}} c_{0}\left|\mathbf{y}_{m \alpha}(k)\right|^{2}
\end{aligned}
$$

which further implies that

$$
\begin{aligned}
\mathbb{E}\left[\hat{V}\left(\mathbf{y}_{k+1}, k+1, r(k+1)\right)\right] & -\mathbb{E}\left[\hat{V}\left(\mathbf{y}_{k}, k, r(k)\right)\right] \\
\leq & \sum_{1 \leq m<\alpha \leq n_{0}} c_{0} \mathbb{E}\left[\left|\mathbf{y}_{m \alpha}(k)\right|^{2}\right] .
\end{aligned}
$$

Similarly to the proof of Theorem 1 , we can derive that $\lim _{k \rightarrow+\infty} \mathbb{E}\left[\left|\mathbf{y}_{m \alpha}(k)\right|^{2}\right]=0\left(1 \leq m<\alpha \leq n_{0}\right)$, namely, $\lim _{k \rightarrow+\infty}$ $\mathbb{E}\left[\left|y_{m}(k)-y_{\alpha}(k)\right|^{2}\right]=0\left(1 \leq m<\alpha \leq n_{0}\right)$. The proof of this theorem is complete.

Remark 6: In Theorem 2, LMI-based conditions have been obtained that ensure the coupled system (1) of $n_{0}$ identical neural networks to be asymptotically synchronized in the mean square, and the criterion is expressed in terms of the solution to a set of LMIs that can be effectively solved by LMI toolbox. We mention here that the mean square exponential synchronization can also be guaranteed under the same conditions using the method developed in [22]. The reason we consider the asymptotic synchronization is to avoid unnecessarily complicated mathematical derivations.

\section{NUMERICAL EXAMPLES}

In this section, two examples are presented to demonstrate the effectiveness of our main results.

Example 1: Consider a three-neuron neural network (1) with the following parameters:

$$
\begin{array}{rlrl}
D(1) & =\left[\begin{array}{ccc}
0.8 & 0 & 0 \\
0 & 0.3 & 0 \\
0 & 0 & 0.5
\end{array}\right] & A(1)=\left[\begin{array}{ccc}
0.2 & -0.5 & 0.1 \\
0.2 & -0.1 & 0 \\
0 & -0.1 & 0.2
\end{array}\right] \\
B(1) & =\left[\begin{array}{ccc}
0.1 & 0.1 & 0.2 \\
0.2 & -0.2 & 0 \\
0.2 & -0.1 & -0.1
\end{array}\right] \quad C(1)=\left[\begin{array}{ccc}
0.2 & 0.2 & -0.1 \\
0 & 0.4 & 0.3 \\
-0.3 & 0 & 0.2
\end{array}\right] \\
D(2) & =\left[\begin{array}{ccc}
0.8 & 0 & 0 \\
0 & 0.1 & 0 \\
0 & 0 & 0.6
\end{array}\right] \quad A(2)=\left[\begin{array}{ccc}
0.8 & -0.2 & 0.1 \\
0.1 & -0.1 & 0.2 \\
0.1 & 0 & 0.2
\end{array}\right] \\
B(2) & =\left[\begin{array}{ccc}
0.2 & 0.1 & 0.1 \\
0.2 & -0.2 & 0 \\
0.1 & -0.1 & -0.1
\end{array}\right] \quad C(2)=\left[\begin{array}{ccc}
0.2 & -0.1 & 0.1 \\
0.1 & 0.2 & 0.3 \\
0.8 & 0 & 0.2
\end{array}\right] \\
\Pi & =\left[\begin{array}{ll}
0.6 & 0.4 \\
0.7 & 0.3
\end{array}\right] \\
\tau_{1,1} & =7 & &
\end{array}
$$

Take the activation functions as follows:

$$
\begin{aligned}
& f_{1}(s)=g_{1}(s)=h_{1}(s)=-\tanh (0.6 s) \\
& f_{2}(s)=g_{2}(s)=h_{2}(s)=\tanh (0.4 s) \\
& f_{3}(s)=g_{3}(s)=h_{3}(s)=0.6 \tanh (s) .
\end{aligned}
$$

It is easy to see that $\Lambda_{1}=\Sigma_{1}=\Upsilon_{1}=0$ and $\Lambda_{2}=$ $\Sigma_{2}=\Upsilon_{2}=\operatorname{diag}\{-0.3,0.2,0.3\}$. With the above parameters, by using Matlab LMI toolbox, we solve the LMIs (9) and obtain the feasible solution as follows:

$$
\begin{aligned}
P_{1} & =\left[\begin{array}{lll}
32.5589 & -4.4445 & -5.7962 \\
-4.4445 & 61.8387 & -9.5629 \\
-5.7962 & -9.5629 & 33.0501
\end{array}\right] \\
P_{2} & =\left[\begin{array}{lll}
38.4776 & -4.9491 & -3.2703 \\
-4.9491 & 64.3330 & -8.2671 \\
-3.2703 & -8.2671 & 56.3423
\end{array}\right] \\
Q & =\left[\begin{array}{ccc}
58.6666 & -0.6842 & 0.2396 \\
-0.6842 & 58.0232 & 0.9247 \\
0.2396 & 0.9247 & 57.4604
\end{array}\right] \\
R & =\left[\begin{array}{ccc}
21.7110 & 0 & 0 \\
0 & 16.2706 & 0 \\
0 & 0 & 16.4010
\end{array}\right] \\
\Omega_{1} & =\operatorname{diag}\{87.7420,101.0563,83.8544\} \\
\Omega_{2} & =\operatorname{diag}\{116.1459,84.9059,86.7772\} \\
\Theta_{1} & =\Theta_{2}=\operatorname{diag}\{155.3972,154.4722,153.5926\} \\
\Delta_{1} & =\operatorname{diag}\{214.7684,188.3660,185.0105\} \\
\Delta_{2} & =\operatorname{diag}\{268.4250,199.1742,203.5137\} .
\end{aligned}
$$

$$
\hat{\xi}_{m \alpha}(k, i)=\left[\begin{array}{llllll}
\mathbf{y}_{m \alpha}^{T}(k) & \mathbf{f}_{m \alpha}^{T}(k) & \mathbf{g}_{m \alpha}^{T}(k) & \mathbf{g}_{m \alpha}^{T}\left(k-\tau_{1, i}\right) & \mathbf{h}_{m \alpha}^{T}(k) & \hat{\mathbf{h}}_{m \alpha}^{T}(k, i)
\end{array}\right]^{T} .
$$



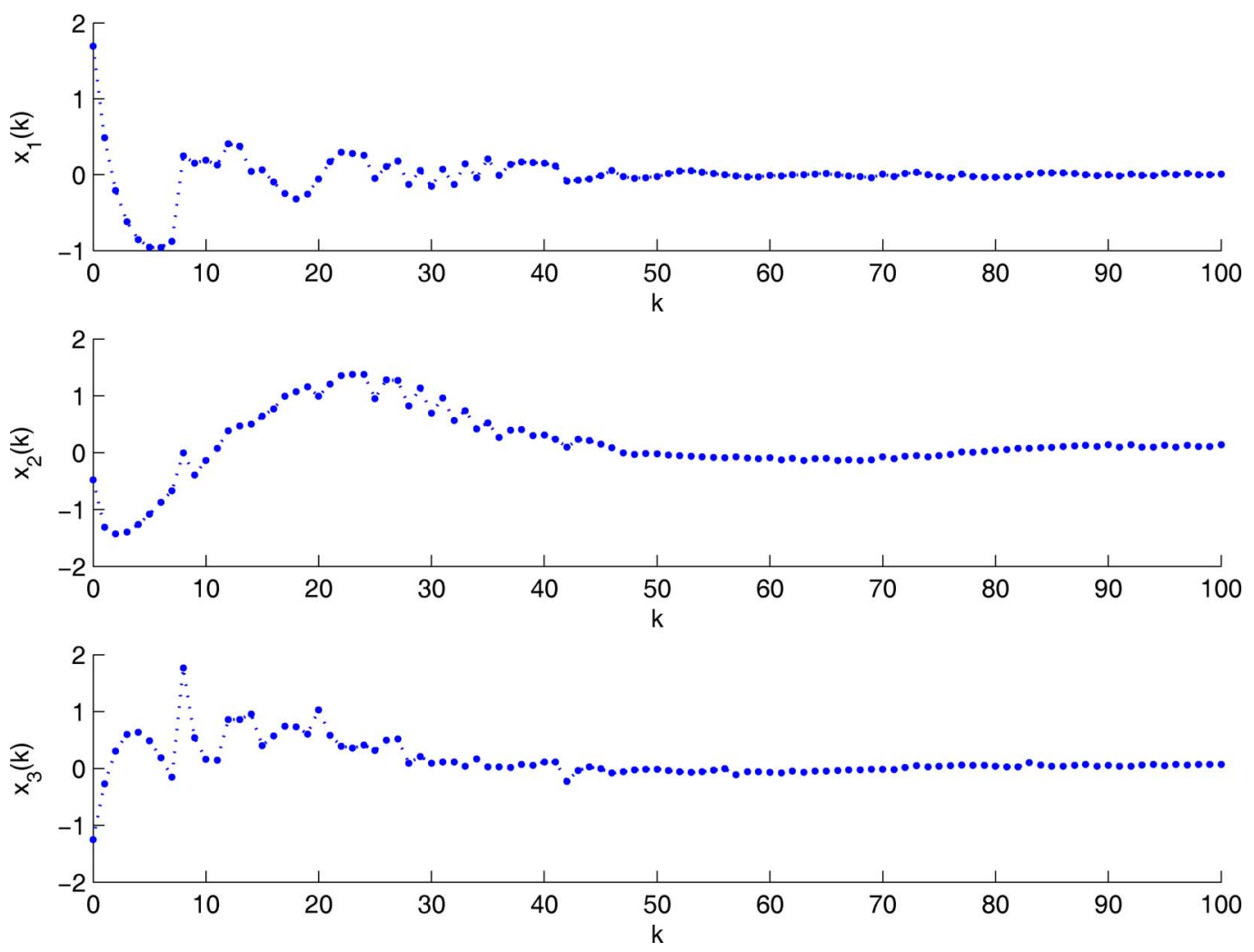

Fig. 1. Evolution of state.

From Theorem 1, it follows that the delayed mode-dependent Markovian neural network (1) with the given parameters is globally asymptotically stable in mean square. The numerical simulation further verifies this result (see Fig. 1).

Example 2: Now, consider the coupled neural network (35) of multiple identical mode-dependent Markovian neural networks. For simplicity, we take $n=2$ and $n_{0}=3$. Other parameters are given as follows:

$$
\begin{aligned}
& D(1)=\left[\begin{array}{cc}
1.7 & 0 \\
0 & 0.3
\end{array}\right] \quad A(1)=\left[\begin{array}{cc}
2 & -0.2 \\
0.2 & -1
\end{array}\right] \\
& B(1)=\left[\begin{array}{cc}
0.8 & 0.6 \\
0.2 & -0.2
\end{array}\right] \quad C(1)=\left[\begin{array}{cc}
2 & 0.2 \\
-0.3 & 0
\end{array}\right] \\
& D(2)=\left[\begin{array}{cc}
0.8 & 0 \\
0 & 1.1
\end{array}\right] \quad A(2)=\left[\begin{array}{cc}
3 & -0.2 \\
0.2 & -1
\end{array}\right] \\
& B(2)=\left[\begin{array}{ll}
1.2 & 0.1 \\
0.2 & -2
\end{array}\right] \quad C(2)=\left[\begin{array}{cc}
2 & -0.1 \\
1 & 0.2
\end{array}\right] \\
& W=\left[\begin{array}{ccc}
-0.2 & 0.1 & 0.1 \\
0.1 & -0.2 & 0.1 \\
0.1 & 0.1 & -0.2
\end{array}\right] \quad \Gamma_{1}=\left[\begin{array}{ll}
1 & 0 \\
0 & 1
\end{array}\right] \\
& \Gamma_{2}=\left[\begin{array}{cc}
1.2 & 0 \\
0 & 0.8
\end{array}\right] \quad \Pi=\left[\begin{array}{ll}
0.6 & 0.4 \\
0.5 & 0.5
\end{array}\right] \\
& \tau_{1,1}=5 \quad \tau_{1,2}=6 \quad \tau_{2,1}=3 \quad \tau_{2,2}=5 \text {. }
\end{aligned}
$$

In addition, the activation functions are given as follows:

$$
\begin{aligned}
& f_{1}(s)=g_{1}(s)=h_{1}(s)=\tanh (-0.6 s) \\
& f_{2}(s)=g_{2}(s)=h_{2}(s)=0.4 \tanh (s) .
\end{aligned}
$$

It is easy to check that

$\Lambda_{1}=\Sigma_{1}=\Upsilon_{1}=\left[\begin{array}{ll}0 & 0 \\ 0 & 0\end{array}\right] \quad \Lambda_{2}=\Sigma_{2}=\Upsilon_{2}=\left[\begin{array}{cc}-0.3 & 0 \\ 0 & 0.2\end{array}\right]$.

With the above parameters, by using Matlab LMI toolbox, we can find the feasible solutions to the LMIs (36) as follows:

$$
\begin{aligned}
P_{1} & =\left[\begin{array}{cc}
0.0322 & -0.0004 \\
-0.0004 & 0.0416
\end{array}\right] \\
P_{2} & =\left[\begin{array}{cc}
0.0009 & -0.0032 \\
-0.0032 & 0.0877
\end{array}\right] \\
Q & =\left[\begin{array}{cc}
3.9792 & -0.0868 \\
-0.0868 & 4.6873
\end{array}\right] \\
R & =\left[\begin{array}{ll}
1.9355 & 0.0184 \\
0.0184 & 0.9509
\end{array}\right] \\
\Omega_{121} & =\operatorname{diag}\{4.5739,4.2880\} \\
\Omega_{122} & =\operatorname{diag}\{4.4640,4.7110\} \\
\Omega_{131} & =\operatorname{diag}\{4.5739,4.2880\} \\
\Omega_{132} & =\operatorname{diag}\{4.6647,4.3617\} \\
\Omega_{231} & =\operatorname{diag}\{4.5739,4.2880\} \\
\Omega_{232} & =\operatorname{diag}\{4.4641,4.3159\} \\
\Theta_{121} & =\operatorname{diag}\{5.4734,5.7307\} \\
\Theta_{122} & =\operatorname{diag}\{5.4734,5.7307\} \\
\Theta_{131} & =\operatorname{diag}\{5.4734,5.7307\} \\
\Theta_{132} & =\operatorname{diag}\{5.4734,5.7307\} \\
\Theta_{231} & =\operatorname{diag}\{5.4734,5.7307\} \\
\Theta_{232} & =\operatorname{diag}\{5.4734,5.7307\}
\end{aligned}
$$



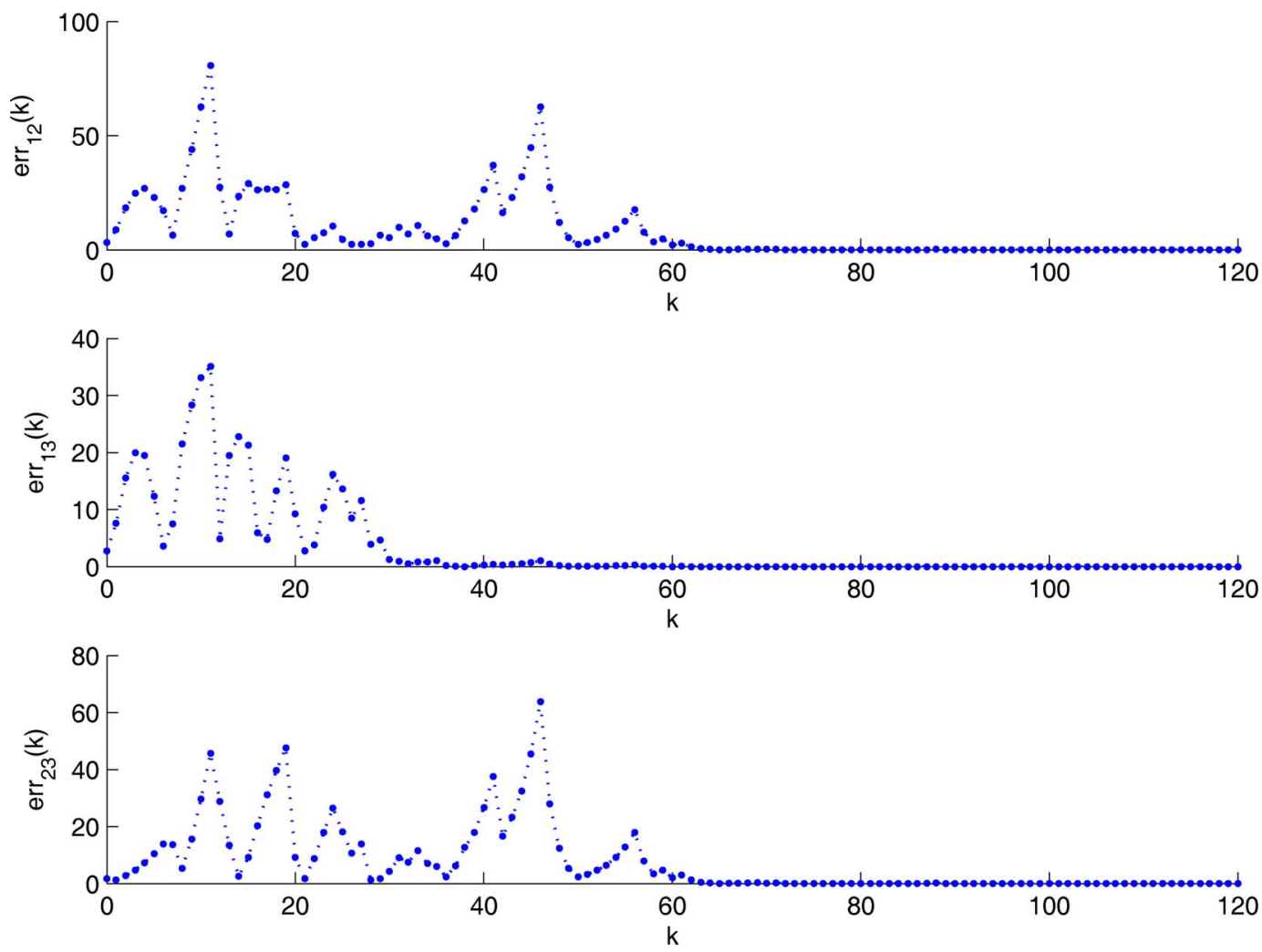

Fig. 2. Evolution of synchronization error.

$$
\begin{aligned}
& \Delta_{121}=\operatorname{diag}\{16.5744,9.6779\} \\
& \Delta_{122}=\operatorname{diag}\{20.7670,11.6500\} \\
& \Delta_{131}=\operatorname{diag}\{16.5744,9.6779\} \\
& \Delta_{132}=\operatorname{diag}\{20.7670,11.6500\} \\
& \Delta_{231}=\operatorname{diag}\{16.5744,9.6779\} \\
& \Delta_{232}=\operatorname{diag}\{20.7670,11.6500\} .
\end{aligned}
$$

Then, it follows from Theorem 2 that the coupled Markovian neural network (35) with the given parameters is synchronized in the mean square. The numerical simulation shows that the synchronization error approaches zero. In Fig. 2, $\operatorname{err}_{12}(k)=$ $\left|y_{1}(k)-y_{2}(k)\right|, \operatorname{err}_{13}(k)=\left|y_{1}(k)-y_{3}(k)\right|$, and $\operatorname{err}_{23}(k)=$ $\left|y_{2}(k)-y_{3}(k)\right|$.

\section{CONCLUSION}

In this paper, we have introduced a new class of DNNs with Markovian jumping parameters as well as mode-dependent mixed time delays. We have first dealt with the stability analysis problem of the addressed neural networks. A special inequality has been developed to account for the mixed time delays in the discrete-time setting, and a novel Lyapunov-Krasovskii functional has been put forward to reflect the mode-dependent time delays. Sufficient conditions have been established in terms of LMIs that guarantee the stochastic stability. We have then turned to the synchronization problem among an array of identical coupled Markovian jumping neural networks with mixed mode-dependent time delays. By utilizing the Lyapunov stability theory and the Kronecker product, it has been shown that the addressed synchronization problem is solvable if several LMIs are feasible. A unified LMI approach has been developed to solve the stability analysis and synchronization problems of the class of neural networks under investigation, where the LMIs can be easily solved by using the available Matlab LMI toolbox. Two numerical examples have been presented to illustrate the usefulness and effectiveness of the main results obtained.

\section{REFERENCES}

[1] S. Arik, "Stability analysis of delayed neural networks," IEEE Trans. Circuits Syst. I, Fund. Theory Appl., vol. 47, no. 7, pp. 1089-1092, Jul. 2000.

[2] Y. Bengio, P. Frasconi, and P. Simard, "The problem of learning longterm dependencies in recurrent networks," in Proc. IEEE Int. Conf. Neural Netw., 1993, vol. 3, pp. 1183-1188.

[3] D. Bolle, P. Dupont, and B. Vinck, "On the overlap dynamics of multistate neural networks with a finite number of patterns," J. Phys. A, Math. Gen., vol. 25, pp. 2859-2872, 1992.

[4] S. Boyd, L. EI Ghaoui, E. Feron, and V. Balakrishnan, Linear Matrix Inequalities in System and Control Theory. Philadelphia, PA: SIAM, 1994.

[5] J. Cao, P. Li, and W. Wang, "Global synchronization in arrays of delayed neural networks with constant and delayed coupling," Phys. Lett. A, vol. 353, pp. 318-325, 2006.

[6] J. Cao and Q. Song, "Stability in Cohen-Grossberg-type bidirectional associative memory neural networks with time-varying delays," Nonlinearity, vol. 19, no. 7, pp. 1601-1617, 2006.

[7] M. P. Casey, "The dynamics of discrete-time computation with application to recurrent neural networks and finite state machine extraction," Neural Comput., vol. 8, no. 6, pp. 1135-1178, 1996.

[8] A. Cleeremans, D. Servan-Schreiber, and J. L. McClelland, "Finite state automata and simple recurrent networks," Neural Comput., vol. 1, no. 3, pp. 372-381, 1989

[9] G. Chen, J. Zhou, and Z. Liu, "Global synchronization of coupled delayed neural networks and applications to chaotic CNN models," Int. J. Bifurc. Chaos, vol. 14, no. 7, pp. 2229-2240, 2004. 
[10] W.-H. Chen, X. Lu, and D.-Y. Liang, "Global exponential stability for discrete-time neural networks with variable delays," Phys. Lett. A, vol. 358, no. 3, pp. 186-198, 2006.

[11] J. L. Elman, "Finding structure in time," Cogn. Sci., vol. 14, pp. 179-211, 1990.

[12] W. Freeman, "Spatial properties of an EEG in the olfactory bulb and olfactory cortex," Electroencephalogr. Clinical Neurophysiol., vol. 44, pp. 586-605, 1978.

[13] H. Gao, J. Lam, and C. Wang, "Robust energy-to-peak filter design for stochastic time-delay systems," Syst. Control Lett., vol. 55, no. 2, pp. 101-111, 2006.

[14] H. Gao, J. Lam, and G. Chen, "New criteria for synchronization stability of general complex dynamical networks with coupling delays," Phys. Lett. A, vol. 360, no. 2, pp. 263-273, 2006.

[15] H. Gao, P. Shi, and J. Wang, "Parameter-dependent robust stability of uncertain time-delay systems," J. Comput. Appl. Math., vol. 206, no. 1, pp. 366-373, 2007.

[16] K. Gopalsamy and X. Z. He, "Delay-independent stability in bi-directional associative memory networks," IEEE Trans. Neural Netw., vol. 5, no. 6, pp. 998-1002, Nov. 1994.

[17] C. Gray, "Synchronous oscillations in neuronal systems: mechanism and functions," J. Comput. Neurosci., vol. 1, pp. 11-38, 1994.

[18] S. Hu and J. Wang, "Global robust stability of a class of discrete-time interval neural networks," IEEE Trans. Circuits Syst. I, Reg. Papers, vol. 53, no. 1, pp. 129-138, Jan. 2006.

[19] J. Liang, J. Cao, and J. Lam, "Convergence of discrete-time recurrent neural networks with variable delay," Int. J. Bifurc. Chaos, vol. 15, no. 2, pp. 581-595, 2005.

[20] J. Liang, J. Cao, and D. W. C. Ho, "Discrete-time bidirectional associative memory neural networks with variable delays," Phys. Lett. A, vol. 335, no. 2-3, pp. 226-234, 2005.

[21] Y. Liu, Z. Wang, and X. Liu, "Global exponential stability of generalized recurrent neural networks with discrete and distributed delays," Neural Netw., vol. 19, pp. 667-675, 2006.

[22] Y. Liu, Z. Wang, and X. Liu, "Robust stability of discrete-time stochastic neural networks with time-varying delays," Neurocomputing, vol. 71, no. 4-6, pp. 823-833, 2008.

[23] Y. Liu, Z. Wang, and X. Liu, "Synchronization and state estimation for discrete-time complex networks with distributed delays," IEEE Trans. Syst. Man Cybern. B, Cybern., vol. 38, no. 5, pp. 1314-1325, Oct. 2008.

[24] W. Lu and T. Chen, "Synchronization of coupled connected neural networks with delays," IEEE Trans. Circuits Syst. I, Reg. Papers, vol. 51, no. 12, pp. 2491-2503, Dec. 2004.

[25] W. Lu and T. Chen, "Synchronization analysis of linearly coupled networks of discrete time systems," Physica D, vol. 198, pp. 148-168, 2004.

[26] W. Lu and T. Chen, "A new approach to synchronization analysis of linearly coupled map lattices," Chin. Ann. Math. B, vol. 28, no. 2, pp. 149-160, 2007.

[27] W. Lu and T. Chen, "Global synchronization of discrete-time dynamical network with a directed graph," IEEE Trans. Circuits Syst. II, Exp. Briefs, vol. 54, no. 2, pp. 136-140, Feb. 2007.

[28] S. Mohamad and K. Gopalsamy, "Exponential stability of continuoustime and discrete-time cellular neural networks with delays," Appl. Math. Comput., vol. 135, no. 1, pp. 17-38, 2003.

[29] S. Mohamad, "Exponential stability preservation in discrete-time analogues of artificial neural networks with distributed delays," J. Comput. Appl. Math., vol. 215, no. 1, pp. 270-287, 2008.

[30] J. C. Principle, J.-M. Kuo, and S. Celebi, "An analysis of the gamma memory in dynamic neural networks," IEEE Trans. Neural Netw., vol. 5, no. 2, pp. 337-361, Mar. 1994.

[31] H. Qiao, J. Peng, and Z. B. Xu, "A reference model approach to stability analysis of neural networks," IEEE Trans. Syst. Man Cybern. B, Cybern., vol. 33, no. 6, pp. 925-936, Dec. 2003.

[32] H. Qiao, J. Peng, and Z. B. Xu, "Nonlinear measures: A new approach to exponential stability analysis for Hopfield-type neural networks," IEEE Trans. Neural Netw., vol. 12, no. 2, pp. 360-370, Mar. 2001.

[33] Y. Shen and J. Wang, "Noise-induced stabilization of the recurrent neural networks with mixed time-varying delays and Markovian-switching parameters," IEEE Trans. Neural Netw., vol. 18, no. 6, pp. 1857-1862, Nov. 2007

[34] Q. Song and J. Cao, "Periodic solutions and its exponential stability of reaction-diffusion recurrent neural networks with continuously distributed delays," Nonlinear Anal.: Real World Appl., vol. 7, no. 1, pp. 65-80, 2006.
[35] A. Stuart and A. Humphries, Dynamical Systems and Numerical Analysis. Cambridge, U.K.: Cambridge Univ. Press, 1996.

[36] D. W. Tank and J. J. Hopfield, "Neural computation by concentrating information in time," in Proc. Nat. Acad. Sci., 1987, vol. 84, pp. 1896-1991

[37] P. Tino, M. Cernansky, and L. Benuskova, "Markovian architectural bias of recurrent neural networks," IEEE Trans. Neural Netw., vol. 15, no. 1, pp. 6-15, Jan. 2004.

[38] Z. Wang, Y. Liu, and X. Liu, "On global asymptotic stability of neural networks with discrete and distributed delays," Phys. Lett. A, vol. 345, no. 4-6, pp. 299-308, 2005.

[39] Z. Wang, Y. Liu, M. Li, and X. Liu, "Stability analysis for stochastic Cohen-Grossberg neural networks with mixed time delays," IEEE Trans. Neural Netw., vol. 17, no. 3, pp. 814-820, May 2006.

[40] Z. Wang, Y. Liu, L. Yu, and X. Liu, "Exponential stability of delayed recurrent neural networks with Markovian jumping parameters," Phys. Lett. A, vol. 356, no. 4-5, pp. 346-352, 2006.

[41] Z. Wang, Y. Liu, K. Fraser, and X. Liu, "Stochastic stability of uncertain Hopfield neural networks with discrete and distributed delays," Phys. Lett. A, vol. 354, no. 4, pp. 288-297, 2006.

[42] C. Wu, "Synchronization in arrays of coupled nonlinear systems with delay and nonreciprocal time-varying coupling," IEEE Trans. Circuits Syst. II, Exp. Briefs, vol. 52, no. 5, pp. 282-286, May 2005.

[43] W. Xiong and J. Cao, "Global exponential stability of discrete-time Cohen-Grossberg neural networks," Neurocomputing, vol. 64, pp. 433-446, 2005.

[44] H. Zhang and Y. Wang, "Stability analysis of Markovian jumping stochastic Cohen-Grossberg neural networks with mixed time delays," IEEE Trans. Neural Netw., vol. 19, no. 2, pp. 366-370, Feb. 2008.

[45] H. Zhao and L. Wang, "Stability and bifurcation for discrete-time Cohen-Grossberg neural network," Appl. Math. Comput., vol. 179, no. 2, pp. 787-798, 2006.

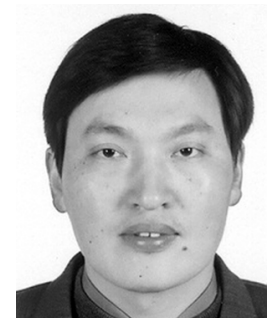

Yurong Liu received the B.Sc. degree in mathematics from Suzhou University, Suzhou, China, in 1986, the M.Sc. degree in applied mathematics from Nanjing University of Science and Technology, Nanjing, China, in 1989, and the $\mathrm{Ph} . \mathrm{D}$. degree in applied mathematics from Suzhou University, Suzhou, China, in 2000

Currently, he is a Professor at the Department of Mathematics, Yangzhou University, Yangzhou, China. His current interests include neural networks, nonlinear dynamics, time-delay systems, and chaotic dynamics.

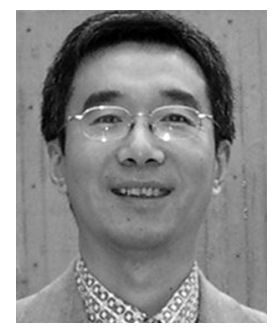

Zidong Wang (SM'03) was born in Jiangsu, China, in 1966. He received the B.Sc. degree in mathematics from Suzhou University, Suzhou, China, in 1986 and the M.Sc. degree in applied mathematics and the $\mathrm{Ph} . \mathrm{D}$. degree in electrical and computer engineering from Nanjing University of Science and Technology, Nanjing, China, in 1990 and 1994, respectively.

Currently, he is a Professor of Dynamical Systems and Computing at Brunel University, Uxbridge, Middlesex, U.K. He has published more than 120 papers in refereed international journals. His research interests include dynamical systems, signal processing, bioinformatics, control theory, and applications.

Prof. Wang is currently serving as an Associate Editor for 12 international journals including the IEEE TRANSACTIONS ON AUTOMATIC CONTROL, the IEEE TRANSACTIONS ON NEURAL NeTWORKS, the IEEE TRANSACTIONS on Signal Processing, the IEEE TRANSACTIONS ON Systems, Man, AND CYBERNETICS-PART C: APPLICATIONS AND REVIEWS and the IEEE TRANSACTIONS ON CONTROL SYSTEMS TECHNOLOGY. 


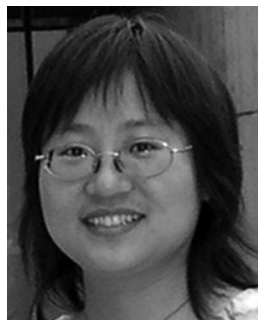

Jinling Liang received the B.Sc. and M.Sc. degrees in mathematics from Northwest University, Xi'an, China, in 1997 and 1999, respectively, and the $\mathrm{Ph} . D$. degree in applied mathematics from Southeast University, Nanjing, China, in 2006.

Currently, she is an Associate Professor at the Department of Mathematics, Southeast University. From January 2004 to March 2004, she was a Research Assistant at the University of Hong Kong. From March to April 2004, she was a Research Assistant at the City University of Hong Kong. From April 2007 to March 2008, she was a Postdoctoral Research Fellow at the Department of Information Systems and Computing, Brunel University, Uxbridge, Middlesex, U.K., sponsored by the Royal Society Sino-British Fellowship Trust Award of the United Kingdom. She has published around 20 papers in refereed international journals. Her research interests include neural networks, complex networks, nonlinear systems, and bioinformatics.

Dr. Liang is an Associate Editor of Neurocomputing, a member of program committee for some international conferences, and a very active reviewer for many international journals.

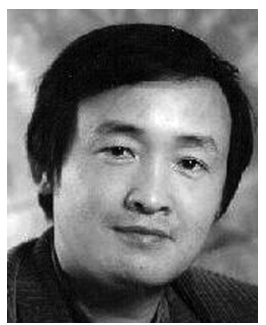

Xiaohui Liu received the B.Eng. degree in computing from Hohai University, Nanjing, China, in 1982 and the Ph.D. degree in computer science from Heriot-Watt University, Edinburg, U.K., in 1988.

Currently, he is a Professor of Computing at Brunel University, Uxbridge, Middlesex, U.K. He leads the Intelligent Data Analysis (IDA) Group, performing interdisciplinary research involving artificial intelligence, dynamic systems, image and signal processing, and statistics, particularly, for applications in biology, engineering, and medicine.

Prof. Liu serves on editorial boards of four computing journals, founded the biennial international conference series on IDA in 1995, and has given numerous invited talks in bioinformatics, data mining, and statistics conferences. 